\title{
Static Aeroelastic Modeling of a Sub-Scale Wind Tunnel Model with Novel Flap Concept
}

\author{
Eric Ting* \\ Stinger Ghaffarian Technologies, Inc., Moffett Field, CA 94035 \\ Nhan Nguyen ${ }^{\dagger}$ \\ NASA Ames Research Center, Moffett Field, CA 94035 \\ Sonia Lebofsky \\ Stinger Ghaffarian Technologies, Inc., Moffett Field, CA 94035
}

\begin{abstract}
This paper examines the static aeroelastic modeling of a flexible wind tunnel model equipped with a novel control effector known as the Variable Camber Continuous Trailing Edge Flap (VCCTEF) system. The wind tunnel model is an approximately $10 \%$ sub-scale version of a wing designed to be equipped on a commercial transport aircraft such as the full-scale NASA Generic Transport Model (GTM). The structure of the model is made highly flexible such that a $10 \%$ of semi-span wing tip deflection is expected at a design lift condition, and a representation of the VCCTEF concept is incorporated on the model. Static aeroelastic modeling is conducted by using a representative single beam structural finite-element model coupled to a vortex-lattice aerodynamic model. The resulting aeroelastic model of the flexible wind tunnel model is compared against experimental wind tunnel test results from the actual model tested at the University of Washington Aeronautical Laboratory (UWAL). Comparison cases are made using reference and VICON measurements of VCCTEF flap deflections from the wind tunnel test data, and percent errors between the lift curve parameters are presented. The results show up to a maximum percent of error of $<10 \%$ with regards to lift curve slope values between the aeroelastic model and the UWAL test data. The agreement of lift curve slope values from the aeroelastic model and experimental test results serves as validation for the coupled vortex-lattice finite-element static aeroelastic model.
\end{abstract}

\section{Introduction}

In recent years, the commercial aircraft industry has been investigating the design of lighter weight wing construction by utilizing breakthroughs made in modern light-weight composites. These wing materials maintain the same load carrying capacity as conventional materials, but result in an overall weight reduction of the aircraft. However, these modern materials generally have reduced structural rigidity in comparison to conventional materials, and the resulting increase in aeroelastic interactions between flight aerodynamics and the wing structure may adversely impact any aerodynamic efficiency improvements due to reduced weight. Development of modeling capabilities of flexible wing aircraft is necessary as these modern wing designs are investigated.

While the increase in flexibility can alter an aircraft's aerodynamics, past research has shown that there exists potential benefits in tailoring a flexible wing structure's aeroelastic shape during flight. In 2010, a conceptual study titled "Elastically Shaped Future Air Vehicle Concept"1 was conducted to investigate the benefits of several advanced aircraft technologies. The results demonstrated that flexible wing systems can utilize active aeroelastic shape control as an avenue by which potential drag savings can be achieved. A novel control surface known as a Variable Camber Continuous Trailing Edge Flap (VCCTEF) system was proposed ${ }^{1,2}$ as a potential control system that could exploit active aeroelastic shape control more efficiently than conventional control surfaces. Under the Fixed Wing Project Active Aeroelastic Shape Control (AASC) element, NASA and Boeing conducted joint studies to investigate the application

\footnotetext{
*Engineer, Intelligent Systems Division, eric.b.ting@ nasa.gov

${ }^{\dagger}$ Research Scientist, Intelligent Systems Division, nhan.t.nguyen@ nasa.gov, AIAA Associate Fellow

‡Engineer, Intelligent Systems Division, sonia.lebofsky@ nasa.gov
} 
of the VCCTEF system ${ }^{3,4}$ on a conventional transport aircraft platform known as the NASA Generic Transport Model (GTM). ${ }^{5}$ The VCCTEF can be utilized to modify the wing's aeroelastic shape, allowing for optimization of the wing's spanwise twist during any point within the flight envelope to achieve a best lift-to-drag (L/D) ratio. The VCCTEF also realizes several other benefits, including the elimination of drag due to trailing edge discontinuities, more efficient lift generation by utilizing camber segments, and the potential for noise reduction.

In 2013, NASA and Boeing worked together with the University of Washington Aeronautical Laboratory (UWAL) to conduct an exploratory wind tunnel test as a proof-of-concept study into the VCCTEF system. A physical model of a sub-scale transport jig-shape wing equipped with a representative VCCTEF system was built and was tested in a low speed wind tunnel. ${ }^{6}$ The wind tunnel test results showed a drag reduction of up to $6.31 \%$ and an improvement in lift-to-drag ratio (L/D) of up to $4.85 \%$ for the configurations tested. ${ }^{7}$ A previous study constructed an aeroelastic model based on a computational fluid dynamic (CFD) Euler code called CART3D that showed agreement with wind tunnel results. ${ }^{8}$

This paper outlines the development and validation of a static aeroelasticity model based upon the sub-scale model of the wing with VCCTEF tested at UWAL. A structural model is developed that uses an equivalent single beam representation of the wind tunnel model. Finite-element $\operatorname{method}^{9-12}$ (FEM) or finite-element analysis (FEA) is used to solve governing beam static equations, and the structural model is taken from another study ${ }^{13}$ that is validated against static load test data of the wind tunnel model that was provided by UWAL. Vortex-lattice modeling ${ }^{14}$ (VLM) is used to evaluate quasi-steady aerodynamic loads, and an automated geometry generation tool generates VLM input to close the loop with FEM. The aeroelastic analysis uses an FEM approach similar to past investigations, ${ }^{15-17}$ and the resulting coupled finite-element vortex-lattice static aeroelastic framework has also been applied in past studies. ${ }^{18-20}$ The coupled finite-element vortex-lattice static aeroelastic model of the UWAL wind tunnel model is able to estimate aeroelastic deformations and generate flexible model lift curves that are compared to UWAL experimental test results. The automated geometry generation tool is also able to model VCCTEF deflections on the UWAL model, and thus multiple experimental test result cases are examined. The agreement between the model and wind tunnel results represents a validation study of the aerodynamic modeling of the VCCTEF as well as the aeroelastic framework of the flexible wing model.

\section{Generic Transport Model and Variable Camber Continuous Trailing Edge Flap}

The NASA GTM (Generic Transport Model) is a sub-scale research platform representative of a classic commercial aircraft configuration. It includes a wind tunnel model and a remotely piloted vehicle, and the full-scale aircraft geometry is obtained by scaling the sub-scale model by a ratio of 200:11. The GTM is modeled as a notional singleaisle, mid-size, 200-passenger aircraft representing one of the most common types of short-to-medium range passenger transport aircraft in the commercial aviation section. It has been tested within the 14-foot-by-22-foot wind tunnel test at NASA Langley Research Center and wind tunnel test data is available to validate computation models. Figure 1 is an illustration of the GTM aircraft. 


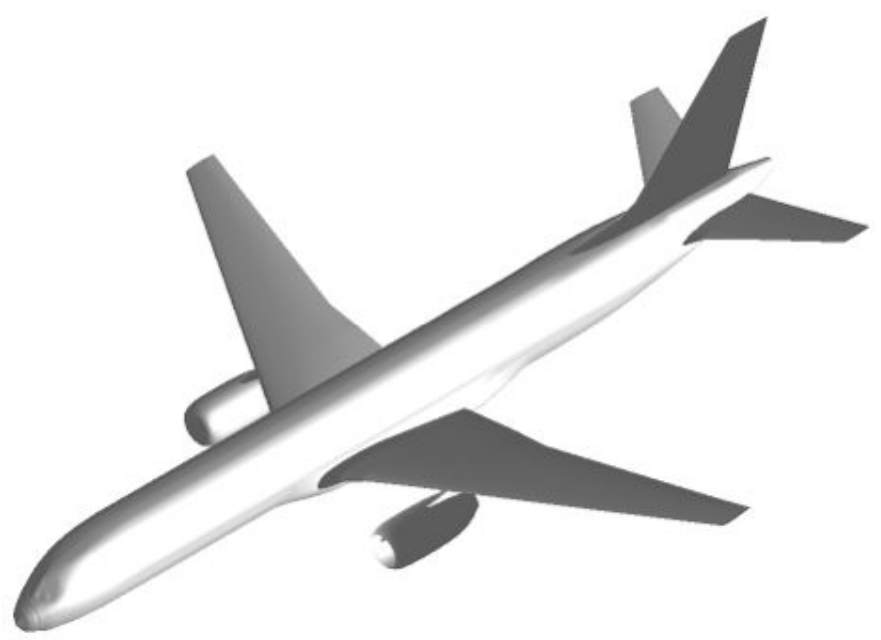

Figure 1. Illustration of GTM Aircraft

The aircraft has a mid-cruise weight of 210,000 lbs for a typical operating load (gear up, flap up) that includes cargo, fuel, and passengers. Fuel weight is about 75,000 lbs for a range of about 3,000 nautical miles. At the design cruise condition of Mach $=0.8$ at $36,000 \mathrm{ft}$, the design lift coefficient is 0.51 .

The GTM is equipped with the VCCTEF (Variable Camber Continuous Trailing Edge Flap) system as a wing shaping control and replacement for conventional ailerons and flaps. Details of the proposed VCCTEF concept are found in references. ${ }^{2-4}$ The VCCTEF is divided into 15 flap segments outboard of the wing break and one flap inboard of the wing break. Each of the outboard segments is 24-inches wide, with the exception of the most outboard, and each possesses three camber segments that can be individually commanded, as shown in Fig. 2.

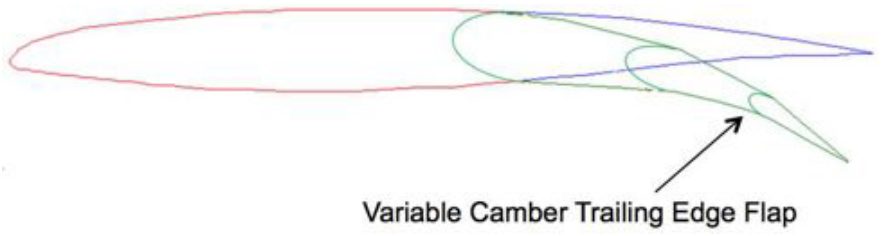

Figure 2. Variable Camber Flap

These camber flaps are joined to the neighboring sections by a flexible and supported material which deforms and provides smooth transitions between flap sections without drag producing gaps. The relative flap deflections between any two adjacent spanwise flap sections are limited due to this flexible skin material. Figure 3 shows the VCCTEF concept deployed on the GTM, where all three camber segments of the inboard flap are shown in light blue, the flexible material connecting neighboring flaps is shown in blue, and the deployed camber segments of the outboard flaps are shown in yellow, orange, and red for the first, second, and third camber segments of each flap, respectively. 


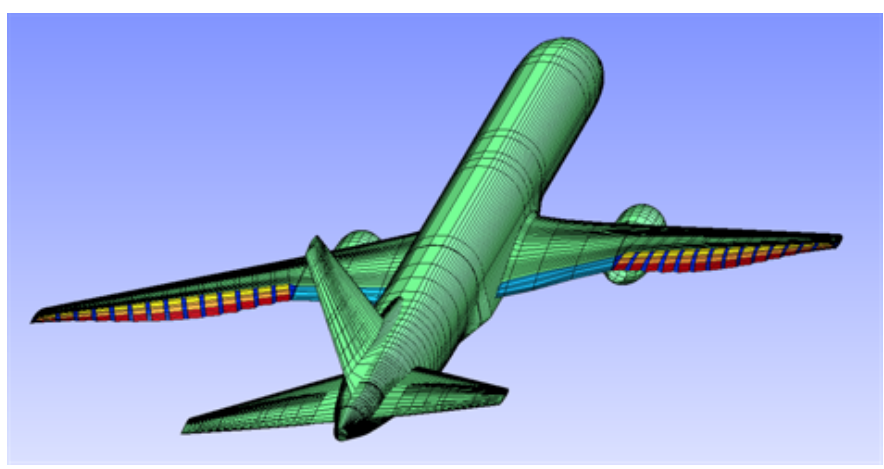

Figure 3. GTM with VCCTEF

\section{Wind Tunnel Model of Flexible Wing}

Designed to be a 10\% sub-scale model of the GTM jig-shape wing, the UWAL (University of Washington Aeronautical Laboratory) wind tunnel model was built and tested in a low-speed wind tunnel as an exploratory assessment of the VCCTEF concept. Designed to have static aeroelastic similarity to a full-scale, modern flexible wing, the UWAL wind tunnel model was constructed such that it would achieve a $10 \%$ of semi-span wing tip deflection when at design lift coefficient of $C_{L}=0.51$. Details of the construction of the UWAL wind tunnel model are referenced. ${ }^{6}$ The outlining dimensions of the UWAL wind tunnel model are shown in Fig. 4.

A representation of the VCCTEF concept is incorporated on the outboard portion of the UWAL wind tunnel model, where 15 outboard sections on the GTM are reduced to 5. Each flap section, however, still maintains three chordwise segments preserving the continuous camber design. The dimensions of the VCCTEF representation built on the UWAL wind tunnel model are shown in Fig. 5.

The wind tunnel test was conducted with a nominal test dynamic pressure of $q_{\infty}=20$ psf. Collated information summarizing the wind tunnel test results are found in references. ${ }^{6,7}$ Figure 6 is a photo of the model being tested in the UWAL wind tunnel.

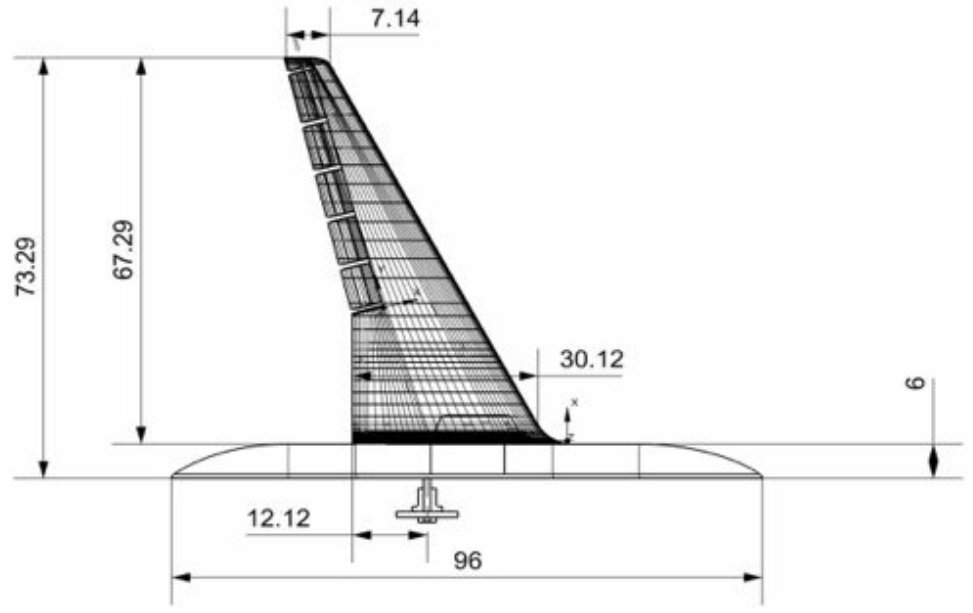

Figure 4. UWAL Wind Tunnel Model with VCCTEF (Courtesy of UWAL) 


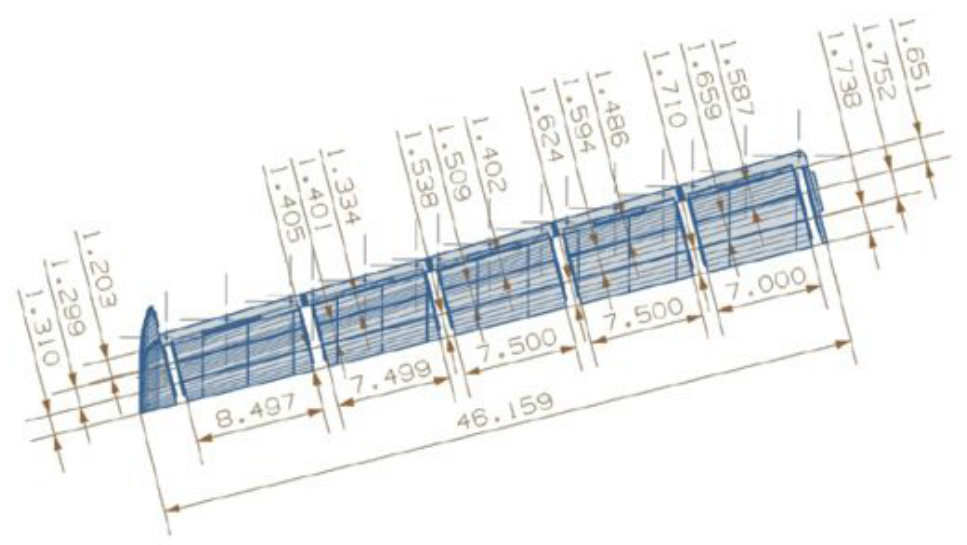

Drawing specifying planform view flap dimensions (measurements in inches)

Figure 5. UWAL Wind Tunnel Model VCCTEF Dimensions (Courtesy of UWAL)

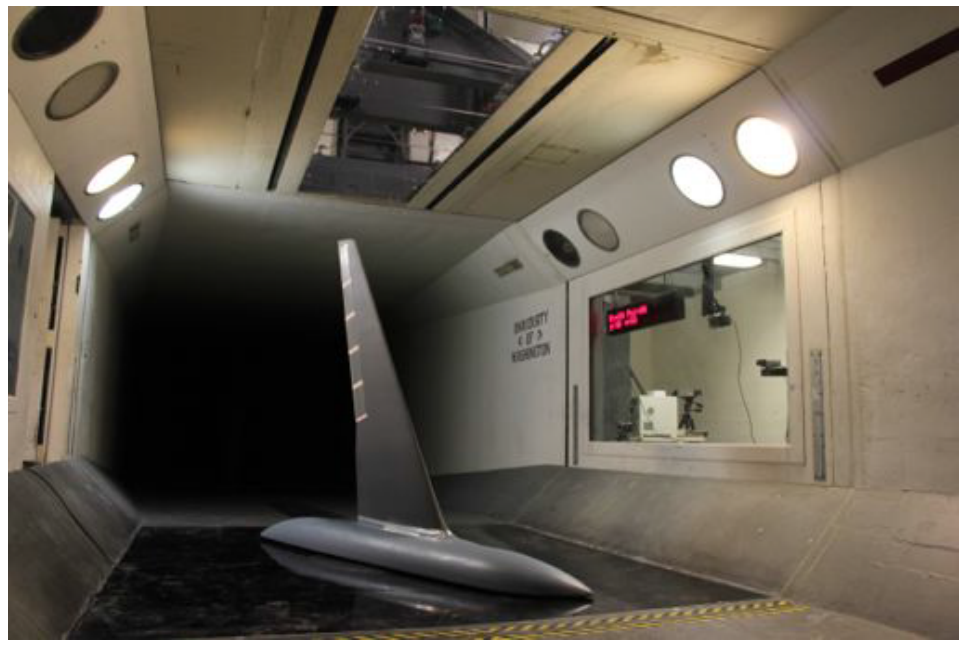

Figure 6. UWAL Wind Tunnel Model in Test Chamber (Courtesy of UWAL)

Table 1 identifies relevant model parameters and scaling between the full-scale GTM and the sub-scale semi-span wind tunnel model.

\begin{tabular}{|c|c|c|}
\hline & Full-Scale GTM & Semi-Span Wind Tunnel Model \\
\hline \hline$M$ & 0.797 & 0.1162 \\
\hline$C_{L}$ & 0.51 & 0.51 \\
\hline$h, \mathrm{ft}$ & 36,000 & 0 \\
\hline$q_{\infty}, \mathrm{psf}$ & 211.09 & 20.00 \\
\hline$S / 2, \mathrm{ft}^{2}$ & 975.5 & 9.638 \\
\hline $\bar{c}, \mathrm{ft}$ & 16.6417 & 1.5963 \\
\hline$b / 2, \mathrm{ft}$ & 62.4167 & 6.1262 \\
\hline
\end{tabular}

Table 1. UWAL Wind Tunnel Model Parameters 


\section{Geometry Generation Tool}

A geometry generation tool is developed in the MATLAB environment to create a package capable of generating geometric surface meshes of the UWAL wind tunnel model. A CAD model was provided by UWAL representing the test model, and cross-sectional data corresponding to the outer mold line of the UWAL model is extracted. Because the mesh is designed for input into VLM, a conformal mesh at component intersections is not needed, though the mesh is made watertight. A symmetric mesh is made representing the UWAL model.

The mesh is built by forming three components: a centerbody, a right wing, and a left wing. The centerbody is formed by 64 cross-sections with 43 points each. Both wings are formed by 46 cross-sections with 424 points each. Figure 7 shows the completed mesh of the baseline UWAL wind tunnel model.

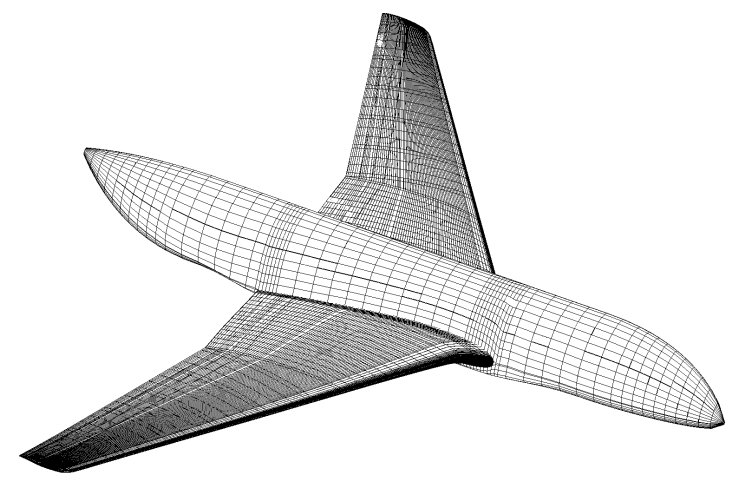

Figure 7. Surface Geometry Mesh of UWAL Wind Tunnel Model

The geometry generation tool is also capable of deflecting the VCCTEF segments on the UWAL wind tunnel model based on a user input. Let the VCCTEF deflection be described by an $n \times m$ matrix where $n$ is the number of camber segments per VCCTEF and $m$ are the number of flap sections. For the wind tunnel model configuration, $n=3$ and $m=5$ such that $\delta=\left[\begin{array}{llll}\delta_{1}^{1} & \delta_{1}^{2} & \ldots & \delta_{1}^{5} \\ \delta_{2}^{1} & \delta_{2}^{2} & \ldots & \delta_{2}^{5} \\ \delta_{3}^{1} & \delta_{3}^{2} & \ldots & \delta_{3}^{5}\end{array}\right]$ where the superscript represents the flap number and the subscript number represents the camber segment. Given a value of deflection $\delta$, the geometry generation tool is able to create a surface mesh representation of the UWAL model with deflected VCCTEF as shown in Fig. 8 (Note that the deflection is exaggerated and the constraint due to the flexible connecting material between flap sections is ignored only for visualization purposes).

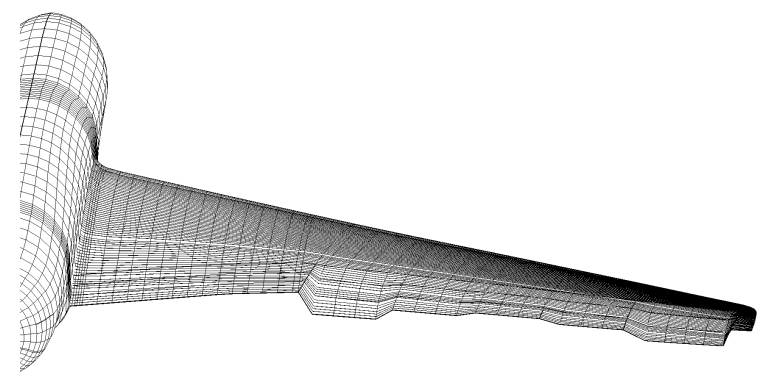

Figure 8. Surface Geometry Mesh of UWAL Wind Tunnel Model Wing with Arbitrary VCCTEF Deflection

The geometry generation tool is capable of generating a deformed mesh based on an input aeroelastic deformation. Let a global coordinate axis frame be represented by $[x, y, z]$. The $x$-axis runs directly aft towards the tail, the $y$-axis runs outboard towards the tip of the right wing, and the $z$-axis runs vertically upwards as shown in Fig. 9. The nose of the centerbody is located at $x_{V}=0 \mathrm{ft}, y_{V}=0 \mathrm{ft}$, and $z_{V}=-0.0823 \mathrm{ft}$. 


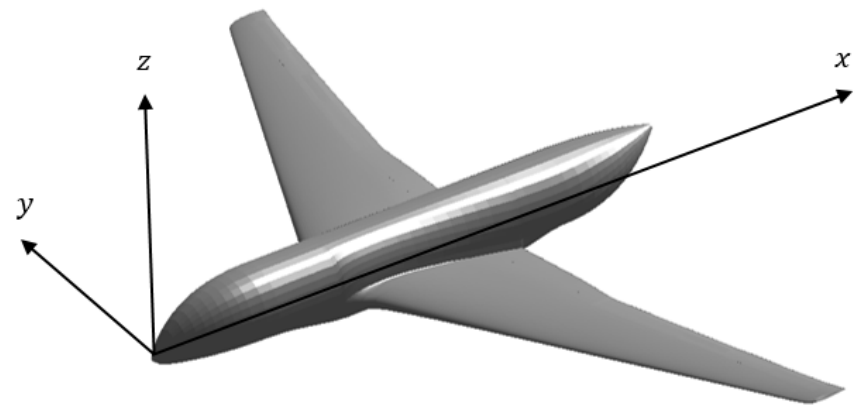

Figure 9. Global Coordinate System

Let the unit vectors $\hat{\mathbf{i}}, \hat{\mathbf{j}}$, and $\hat{\mathbf{k}}$ lie in the directions of the $x$-, $y$-, and $z$ - global directions. An aeroelastic deformation is input into the geometry generation tool as a rotation vector and a translation vector.

$$
\begin{gathered}
\Delta \mathbf{r}=\Delta x \hat{\mathbf{i}}+\Delta y \hat{\mathbf{j}}+\Delta z \hat{\mathbf{k}} \\
\Delta \phi=\Delta \phi_{x} \hat{\mathbf{i}}+\Delta \phi_{y} \hat{\mathbf{j}}+\Delta \phi_{z} \hat{\mathbf{k}}
\end{gathered}
$$

Given a set of undeformed input wing coordinates $\left(x_{w}, y_{w}, z_{w}\right)$ in the global coordinate frame and the definition of an elastic axis location, $\left(x_{e a}, y_{e a}, z_{e a}\right)$, aeroelastic deformation is incorporated by a series of rotations and a set of translations.

$$
\begin{aligned}
\left\{\begin{array}{c}
\tilde{x}_{w} \\
\tilde{y}_{w} \\
\tilde{z}_{w}
\end{array}\right\} & =\left[\begin{array}{ccc}
\cos \Delta \phi_{z} & -\sin \Delta \phi_{z} & 0 \\
\sin \Delta \phi_{z} & \cos \Delta \phi_{z} & 0 \\
0 & 0 & 1
\end{array}\right]\left[\begin{array}{ccc}
1 & 0 & 0 \\
0 & \cos \Delta \phi_{x} & -\sin \Delta \phi_{x} \\
0 & \sin \Delta \phi_{x} & \cos \Delta \phi_{x}
\end{array}\right]\left[\begin{array}{ccc}
\cos \Delta \phi_{y} & 0 & \sin \Delta \phi_{y} \\
0 & 1 & 0 \\
-\sin \Delta \phi_{y} & 0 & \cos \Delta \phi_{y}
\end{array}\right]\left\{\begin{array}{c}
x_{w}-x_{e a}\left(y_{w}\right) \\
0 \\
z_{w}-z_{e a}\left(y_{w}\right)
\end{array}\right\} \\
& +\left\{\begin{array}{c}
x_{e a}\left(y_{w}\right)+\Delta x \\
y_{w}+\Delta y \\
z_{e a}\left(y_{w}\right)+\Delta z
\end{array}\right\}
\end{aligned}
$$

where $\tilde{x}_{w}, \tilde{y}_{w}$, and $\tilde{z}_{w}$ is the location of the deformed outer mold point.

\section{Vortex-Lattice Aerodynamic Model}

VORVIEW/VORLAX is a computational tool used for aerodynamic modeling of aircraft configurations using vortex-lattice method. ${ }^{14}$ Based on lifting line/vortex-lattice aerodynamic theory, VORVIEW is a computationally efficient method for estimating quasi-steady aircraft aerodynamics. Geometric input vehicle configurations are constructed within VORVIEW, which are then discretized into a vortex-lattice input as a series of mean camber panels. Vortex-lattice method code VORLAX then represents the panels with the placement of spanwise and chordwise locations of bound or horseshoe vortices. VORVIEW/VORLAX can then rapidly analyze the total aircraft coefficients, and local sectional data can be post-processed from VORLAX panel pressure data.

VORVIEW is considered a medium-fidelity tool, and limitations associated with vortex-lattice modeling in general apply to VORVIEW/VORLAX aerodynamic analysis. Based on potential flow theory for inviscid and incompressible flow, the drag prediction by VORLAX is most reliable only for induced drag prediction due to the inviscid nature of any vortex-lattice method. Prediction of viscous drag due to boundary layer separation and wave drag due to shockinduced boundary layer separation are generally not conducted by vortex-lattice, and viscous drag estimates must be done using other methods and then added to VORLAX results. VORLAX accounts for the compressibility effect on the aerodynamic coefficients by utilizing a compressibility correction, such as the Prandtl-Glauert correction.

VORVIEW/VORLAX results have been validated by both wind tunnel data ${ }^{5}$ as well as the NASA Cart3D tool, ${ }^{21}$ a high-fidelity inviscid (Euler) CFD analysis code. Agreement between VORVIEW/VORLAX, Cart3D, and wind tunnel results have been shown for the GTM platform. ${ }^{1,2,20}$ In general, both VORVIEW and Cart3D seem to have similar predictive capabilities when compressibility is not a factor. 
Figure 10 shows a panel/polygon discretization of the UWAL wind tunnel model geometry within VORVIEW. A symmetric model of the wind tunnel model is analyzed in VLM, and the reference parameters in Table 1 are adjusted to reflect the full-span model. The geometry generation tool places vortex-panel/polygon edges along the trailing edge locations of the boundaries of the VCCTEF sections. This ensures that deflection of the VCCTEF is fully captured within vortex-lattice analysis even as the wing is aeroelastically changing shape. Because it is not accurately represented as a mean camber lifting surface, the centerbody of the UWAL wind tunnel model is represented using an equivalent flat plate for this study.

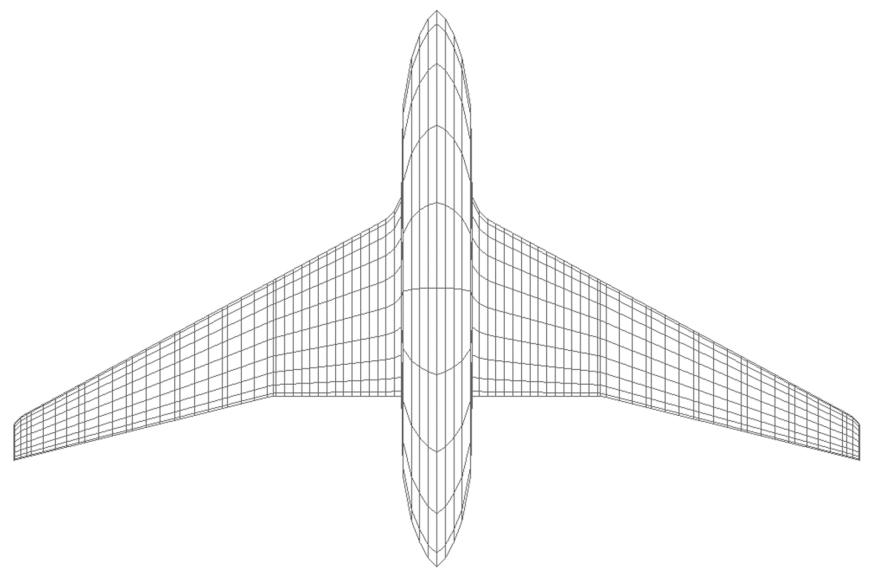

Figure 10. UWAL Wind Tunnel Model Vortex-Lattice Panel/Polygon Discretization in VORVIEW

A flat plate skin friction drag correction is added to VORLAX resulting in an adjustment to estimate the effects of viscous drag.

\section{Structural Finite-Element Model}

Both a detailed 3D NASTRAN model as well as an equivalent beam-stick NASTRAN model were provided by UWAL in addition to the wind tunnel test results. The 3D NASTRAN model demonstrated good agreement with static load test data on the test model. ${ }^{6}$ It is desired to utilize a single straight equivalent beam representation for the elastic axis of the UWAL flexible wing tunnel model, which is a common approach in examining high aspect ratio flexible wings. The equivalent straight beam model utilized in this study was developed in another study ${ }^{13}$ based on optimization of stiffness distributions using static deflections test results.

The equivalent beam model is a single straight beam with no dihedral $\Gamma=0^{\circ}$ and and elastic axis sweep of $\Lambda=20.8299^{\circ}$. Figure 11 represents the vertical bending stiffness $E I_{y y}$ and the torsional stiffness $G J$ along the global spanwise direction of the flexible UWAL wind tunnel model wing. 

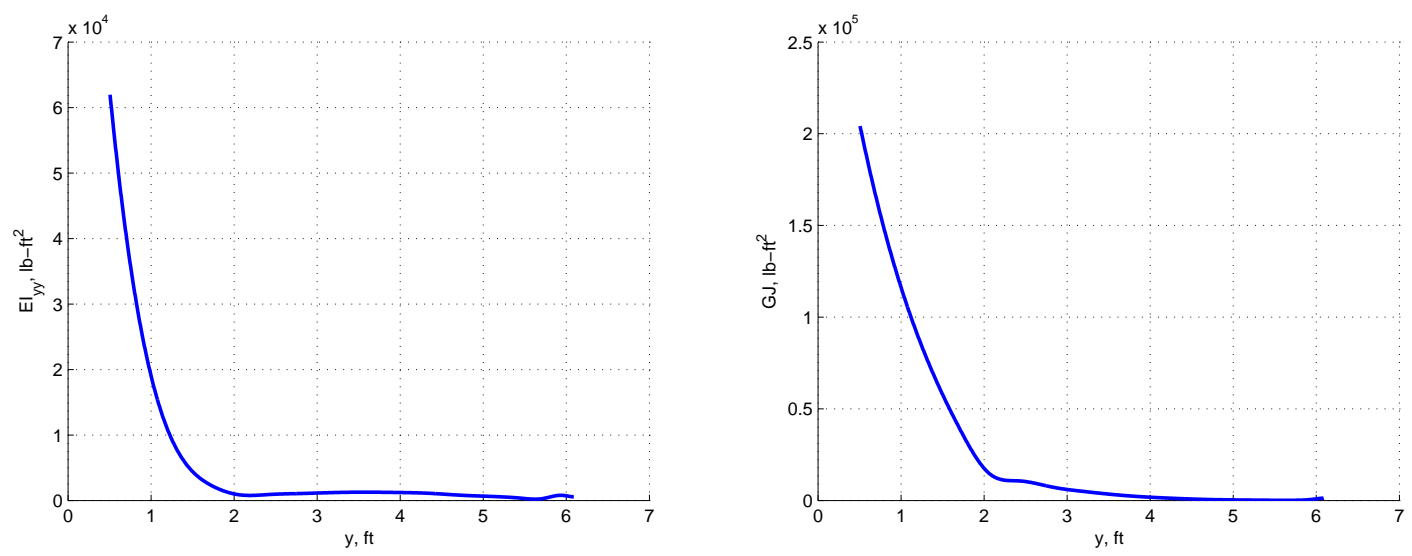

Figure 11. UWAL Wind Tunnel Model Equivalent Beam Bending Stiffness (left) and Torsional Stiffness (Right)

Finite-element analysis is utilized to formulate a structural model of the equivalent straight beam. The analysis is similar to past studies. ${ }^{16-19}$ For an arbitrary straight beam representation of an elastic axis, let a local coordinate system be represented by the coordinate system $[\mu, v, \xi]$ where the $\mu$-axis lies along the beam axis positive towards the tip, the $v$-axis is perpendicular to the beam axis positive pointing forwards, and the $\xi$-axis is perpendicular to the beam axis positive up. The coordinate frames are related through the elastic axis sweep angle $\Lambda$ and a dihedral angle $\Gamma$. Figure 12 shows the relation between the global coordinate system and a local beam coordinate frame.

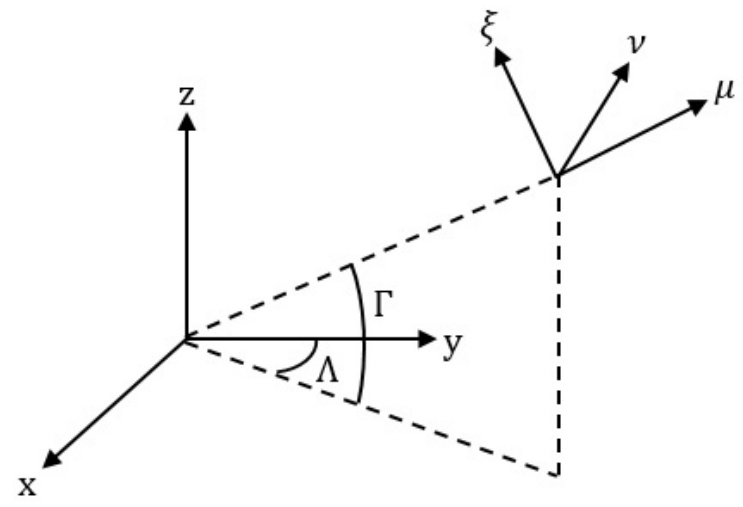

Figure 12. Global and Local Elastic Axis Beam Coordinate System

A series of coordinate transformations can be determined relating a local beam axis to the global axis. The first coordinate transformation rotates the global coordinate system to account for the sweep $\Lambda$, and a second rotation accounts for the dihedral $\Gamma$.

$\left\{\begin{array}{l}\mu \\ v \\ \xi\end{array}\right\}=\left[\begin{array}{ccc}\cos \Gamma & 0 & \sin \Gamma \\ 0 & 1 & 0 \\ -\sin \Gamma & 0 & \cos \Gamma\end{array}\right]\left[\begin{array}{ccc}\sin \Lambda & \cos \Lambda & 0 \\ -\cos \Lambda & \sin \Lambda & 0 \\ 0 & 0 & 1\end{array}\right]\left\{\begin{array}{l}x \\ y \\ z\end{array}\right\}=\left[\begin{array}{ccc}\sin \Lambda \cos \Gamma & \cos \Lambda \cos \Gamma & \sin \Gamma \\ -\cos \Lambda & \sin \Lambda & 0 \\ -\sin \Lambda \text { sin } \Gamma & -\cos \Lambda \sin \Gamma & \cos \Gamma\end{array}\right]\left\{\begin{array}{l}x \\ y \\ z\end{array}\right\}$

With respect to a local beam axis system, the sign conventions for the forces and moments acting on the beam element are defined. Forces are defined positive when they act in the positive directions of the axis system. On a right wing beam element airfoil, moments are defined such that the torsional moment $M_{x}$ is positive in the $\mu$-axis direction, creating a nose-up twist. The flapwise bending moment $M_{y}$ is defined as positive where the moment causes compression on the upper surface of the element and tension on the bottom surface, or in the negative $v$-axis direction. The chordwise bending moment $M_{z}$ is defined as positive when it causes compression along the leading edge of 
airfoil beam element and tension along the trailing edge, or in the positive $\xi$-axis direction. The force and moment conventions are shown in Fig. 13.

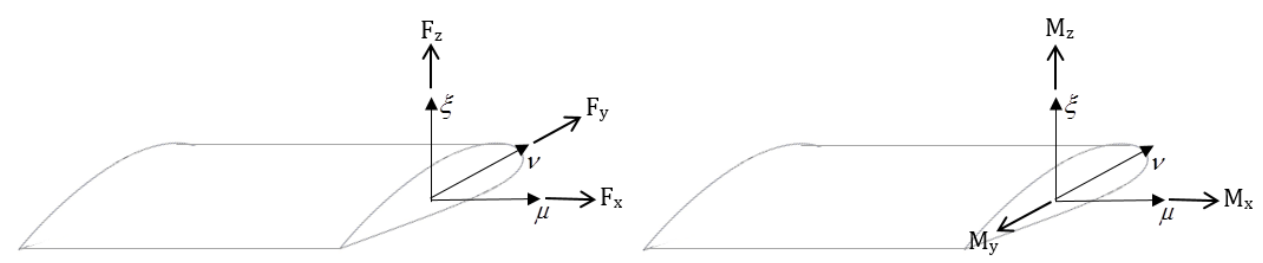

Figure 13. Sign Conventions for Forces and Moments on Local Beam Elements

Per Euler-Bernoulli beam theory, the extensional force, torsional moment, and two bending moments acting on a beam element are given by

$$
\left\{\begin{array}{c}
F_{x} \\
M_{x} \\
M_{y} \\
M_{z}
\end{array}\right\}=\left[\begin{array}{cccc}
E A & 0 & 0 & 0 \\
0 & G J & 0 & 0 \\
0 & 0 & E I_{y y} & -E I_{y z} \\
0 & 0 & -E I_{z y} & E I_{z z}
\end{array}\right]\left\{\begin{array}{c}
\frac{d u}{d x} \\
\frac{d \theta}{d x} \\
\frac{d^{2} w}{d x^{2}} \\
\frac{d^{2} v}{d x^{2}}
\end{array}\right\}
$$

where $u$ is the beam extension, $\theta$ is the beam elastic axis rotation, $w$ is vertical bending (wing out-of-plane or flapwise bending), and $v$ is chordwise bending (wing in-plane bending) with sign conventions shown in Fig. 14. The coupled bending-torsion effect considered in reference ${ }^{22}$ are currently approximated to be negligible.

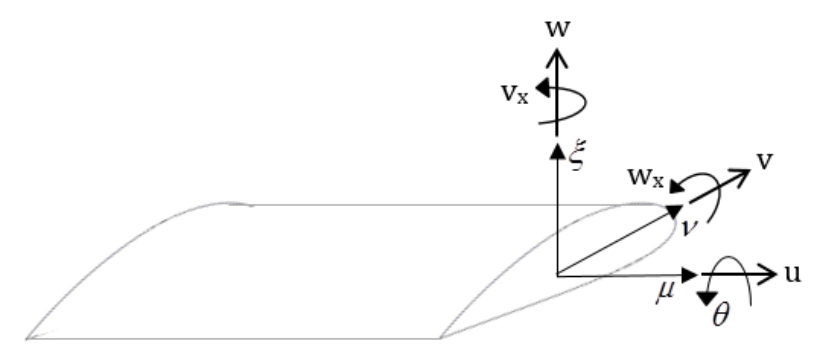

Figure 14. Sign Conventions for Structural Deformation on Local Beam Elements

The force and moment equilibrium equations for the beam element can be determined to be

$$
\begin{gathered}
\frac{\partial F_{x}}{\partial x}+f_{x}=0 \\
\frac{\partial F_{y}}{\partial x}+f_{y}=0 \\
\frac{\partial F_{z}}{\partial x}+f_{z}=0 \\
\frac{\partial M_{x}}{d x}=-m_{x} \\
\frac{\partial^{2} M_{y}}{\partial x^{2}}=f_{z}-\frac{\partial m_{y}}{\partial x} \\
\frac{\partial^{2} M_{z}}{\partial x^{2}}=f_{y}-\frac{\partial m_{z}}{\partial x}
\end{gathered}
$$


For modeling the structural deformation of the flexible UWAL wind tunnel model, only flapwise bending and torsional deformation are considered. Axial extension and chordwise bending are assumed to be negligible in magnitude. Without considering any shear deformation, the governing partial differential equations for the beam deformation result from substitution of Eq. 5 into the equilibrium equations Eqs. 9 and 10 and are given by

$$
\begin{gathered}
\frac{\partial}{\partial x}\left(G J \theta_{x}\right)=-m_{x} \\
\frac{\partial^{2}}{\partial x^{2}}\left(E I_{y y} w_{x x}\right)=f_{z}-\frac{\partial}{\partial x} m_{y}
\end{gathered}
$$

The finite-element approach is used to convert the beam element partial differential equations given above into a set of matrix equations. The wing elastic axis of the UWAL wind tunnel model is broken up in a series of $N$ straight beam elements. For each $n$-th element, the bending and torsional deflections are approximated as

$$
\begin{aligned}
& \Theta_{n}(\mu, t)=\psi_{1}(\mu) \theta_{1_{n}}(t)+\psi_{2}(\mu) \theta_{2_{n}}(t)=\left[\begin{array}{ll}
\psi_{1}(\mu) & \psi_{2}(x)
\end{array}\right]\left[\begin{array}{c}
\theta_{1_{n}}(t) \\
\theta_{2_{n}}(t)
\end{array}\right]=N_{\theta}(\mu) \theta_{n}(t) \\
& W_{n}(\mu, t)=\left[\phi_{1}(\mu) w_{1_{n}}(t)+\phi_{2}(\mu) w_{x, 1_{n}}(t)+\phi_{3}(\mu) w_{2_{n}}(t)+\phi_{4}(\mu) w_{x, 2_{n}}(t)\right] \\
& =\left[\begin{array}{llll}
\phi_{1}(\mu) & \phi_{2}(\mu) & \phi_{3}(\mu) & \phi_{4}(\mu)
\end{array}\right]\left[\begin{array}{c}
w_{1_{n}}(t) \\
w_{x, 1_{n}}(t) \\
w_{2_{n}}(t) \\
w_{x, 2_{n}}(t)
\end{array}\right]=N_{w}(\mu) w_{n}(t)
\end{aligned}
$$

where the subscripts 1 and 2 refer to the start and end nodes for the finite element. The functions $\psi_{j}(\mu), j=1,2$ and $\phi_{k}(\mu), k=1,2,3,4$ are the linear and Hermite polynomial shape functions used as basis functions for approximating the element deformation

$$
\begin{gathered}
\psi_{1}(\mu)=1-\frac{\mu}{l} \\
\psi_{2}(\mu)=\frac{\mu}{l} \\
\phi_{1}(\mu)=1-3\left(\frac{\mu}{l}\right)^{2}+2\left(\frac{\mu}{l}\right)^{3} \\
\phi_{2}(\mu)=l\left[\frac{\mu}{l}-2\left(\frac{\mu}{l}\right)^{2}+\left(\frac{\mu}{l}\right)^{3}\right] \\
\phi_{3}(\mu)=3\left(\frac{\mu}{l}\right)^{2}-2\left(\frac{\mu}{l}\right)^{3} \\
\phi_{4}(\mu)=l\left[-\left(\frac{\mu}{l}\right)^{2}+\left(\frac{\mu}{l}\right)^{3}\right]
\end{gathered}
$$

where $\mu \in[0,1]$ is the local coordinate and $l=\frac{L}{N}$ the $n$-th element length.

The weak-form integral expression can be obtained by pre-multiplying the equilibrium equations Eqs. 12 and 13 by the relevant shape functions and integrating over the length of the element. Integration by parts is applied as needed, and boundary values are released and assumed to be enforced when the global finite-element state space system is formulated.

$$
\begin{gathered}
\sum_{n=1}^{N} \int_{0}^{l}\left(N_{l}^{\prime \mathrm{T}} G J N_{l}^{\prime} \theta\right) d \mu=\sum_{n=1}^{N} \int_{0}^{l} N_{l}^{\mathrm{T}} m_{x} d \mu \\
\sum_{n=1}^{N} \int_{0}^{l}\left(N_{w}^{\prime \prime \mathrm{T}} E I N_{w}^{\prime \prime} w\right) d \mu=\sum_{n=1}^{N} \int_{0}^{l}\left(N_{w}^{\mathrm{T}} f_{z}+N_{w}^{\prime \mathrm{T}} m_{y}\right) d \mu
\end{gathered}
$$

For a simplified analysis of the flexible UWAL wind tunnel model, only sectional lift and pitching moment about the local aerodynamic center are considered for a wing section.

$$
l(y)=c_{l}(y) q_{\infty} c(y)
$$




$$
\begin{aligned}
d(y) & =c_{d}(y) q_{\infty} c(y) \\
m_{a c}(y) & =c_{m_{a c}}(y) q_{\infty} c(y)^{2}
\end{aligned}
$$

The pitching moment about the local aerodynamic center can be transferred to a pitching moment about the local elastic axis node by

$$
m(y)=\left(c_{m_{a c}}(y)+\frac{e(y)}{c(y)} c_{l}(y)\right) q_{\infty} c(y)^{2}
$$

where $e$ is the location of the aerodynamic center relative to the elastic axis along the global $x$-direction, positive when the aerodynamic center is forward of the elastic axis.

The value of the global forces are $f_{Z}=l \cos \alpha+d \sin \alpha$, and $m_{Y}=m$. Utilizing Eq. 4 and setting $\Gamma=0^{\circ}$, the force and moment along the straight elastic axis beam are given by

$$
\begin{gathered}
f_{z}(\mu)=\left(c_{l}(\mu) \cos \alpha+c_{d}(\mu) \sin \alpha\right) q_{\infty} \cos \Lambda c(\mu) \\
m_{x}(\mu)=\left(c_{m_{a c}}(\mu)+\frac{e(\mu)}{c(\mu)} c_{l}(\mu)\right) q_{\infty} \cos ^{2} \Lambda c(\mu)^{2} \\
m_{y}(\mu)=\left(c_{m_{a c}}(\mu)+\frac{e(\mu)}{c(\mu)} c_{l}(\mu)\right) q_{\infty} \tan \Lambda \cos ^{2} \Lambda c(\mu)^{2}
\end{gathered}
$$

where an additional $\cos \Lambda$ term arises from the change in independent variable from the global $y$-axis direction to the $\mu$-axis along the elastic axis beam equivalent.

An element state space system can be formulated from Eqs. 22 and 23 and substitution of Eqs. 27-29. The elemental stiffness and force vectors are given as

$$
\begin{gathered}
K_{n}=\int_{0}^{l}\left[\begin{array}{cc}
N_{w}^{\prime \prime} \mathrm{T} E I_{y y} N_{w}^{\prime \prime} & 0 \\
0 & N_{l}^{\prime \mathrm{T}} G J N_{l}^{\prime} \mathrm{T}
\end{array}\right] d x \\
F_{n}=q_{\infty} \cos \Lambda c \int_{0}^{l}\left[\begin{array}{c}
N_{w}^{\mathrm{T}}\left(c_{l} \cos \alpha+c_{d} \sin \alpha\right)+N_{w}^{\prime \mathrm{T}}\left(c_{m_{a c}}+\frac{e}{c} c_{l}\right) \cos \Lambda c \\
N_{l}^{\mathrm{T}}\left(c_{m_{a c}}+\frac{e}{c} c_{l}\right) \cos \Lambda
\end{array}\right] d x
\end{gathered}
$$

The discrete global approximation system is then formed by enforcing equilibrium conditions at element interfaces and summing each transformed element matrix during the assembly process. The global system stiffness matrix and force vectors are

$$
\begin{gathered}
K=\sum_{n=1}^{N} K_{n} \\
F=\sum_{n=1}^{N} F_{n}
\end{gathered}
$$

and a static matrix equation is of the form

$$
K x_{e}=F
$$

where $x_{e}=\left[\begin{array}{llllllllll}w_{1} & w_{1}^{\prime} & \theta_{1} & w_{2} & w_{2}^{\prime} & \theta_{2} & \ldots & w_{N+1} & w_{N+1}^{\prime} & \theta_{N+1}\end{array}\right]^{\mathrm{T}}$.

Given a solution $W, W_{x}$, and $\Theta$ that can be solved through $x_{e}=K^{-1} F$, a deformation vector can then be determined using the transpose of the matrix in Eq. 4 where the results represents the aeroelastic deformation where

$$
\begin{gathered}
\Delta \mathbf{r}=W \hat{\mathbf{k}} \\
\Delta \phi=\left(\Theta \sin \Lambda+W_{x} \cos \Lambda\right) \hat{\mathbf{i}}+\left(\Theta \cos \Lambda-W_{x} \sin \Lambda\right) \hat{\mathbf{j}}
\end{gathered}
$$

For the flexible UWAL wind tunnel model, the equivalent straight elastic axis beam is discretized into $N=30$ beam elements. 


\section{Static Aeroelasticity Model}

A static aeroelastic model is used in this study in order to predict the aeroelastic wing shape for the flexible UWAL wind tunnel model at the test conditions. A coupled aero-structural static aeroelastic model is used that draws from the coupled vortex-lattice finite-element framework used in previous studies. ${ }^{18-20}$ Figure 15 represents the high level coupling conducted by the iterations in the aeroelastic code.

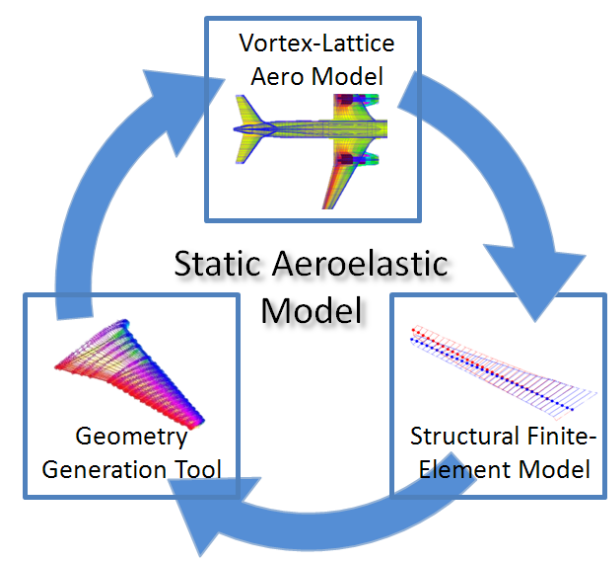

Figure 15. Static Aeroelastic Model Concept

The static aeroelastic code is used to map an input flight condition into the respective static aeroelastic deformed wing shape and corresponding aerodynamic qualities. Given an input Mach number $M$ and altitude $h$, the static aeroelastic code can either accept an input angle of attack $\alpha$ or lift coefficient $C_{L}$. The static aeroelastic solution for the given set $(M, h, \alpha)$ or $\left(M, h, C_{L}\right)$ is thus determined using the following iterative process:

1. Vortex-lattice modeling is conducted on input geometry at the specified flight condition to determine the aircraft total aerodynamic quantities at the input $\alpha$ or $C_{L}$, as well as sectional coefficients $c_{l}(y), c_{d}(y), c_{m_{a c}}(y)$, and $x_{a c}(y)$ or the location of the section aerodynamic centers.

2. The structural FEM model uses the sectional aerodynamic load inputs to calculate the wing's structural deflection. These are represented as a elastic axis twist $\Theta(y)$, flapwise bending deflection $W(y)$ and bending slope $W_{x}(y)$.

3. The FEM solution is converted into deformation vectors $\Delta \mathbf{r}$ and $\Delta \phi$, which are input into the geometry generation tool. The automated geometry tool generates a new aircraft geometry with the deformed wing.

4. Steps 1-3 are repeated until convergence.

The framework above describes a coupled vortex-lattice finite-element model based on coupling of VORLAX-based aerodynamic modeling with the structural FEA model described in the previous section. Implemented in the MATLAB environment, this "VORLAX-FEA" model is the primary static aeroelastic tool used in this study.

Another static aeroelastic model is developed using the same architecture as in Fig. 15 where the structural model is replaced by an equivalent beam model built for NASTRAN static analysis. This model, referred to as the "VORLAXNASTRAN" model, still utilizes VORVIEW/VORLAX as the aerodynamic modeling tool as well as the corresponding geometry generation tool. An identical straight beam model that was used in the VORLAX-FEA model is utilized in the VORLAX-NASTRAN model as a direct comparison between static aeroelastic solutions determined using the MATLAB implemented research based FEA code and NASTRAN based FEM. For the UWAL wind tunnel model's design test condition of $C_{L}=0.51$, static aeroelastic solutions are summarized in Table 2. Data is presented for the right wing where $\Theta$ and $\Delta \phi_{y}$ are positive nose-up. The uncoupled aero-structural results are also included, which can be modeled assuming that the rigid aerodynamic loads are constant and not affected by wing flexibility. 


\begin{tabular}{|c|c|c|c|c|}
\hline & $\alpha(\mathrm{deg})$ & $W_{\text {tip }}(\mathrm{ft})$ & $\Theta_{\text {tip }}(\mathrm{deg}$, positive nose-up) & $\Delta \phi_{y, t i p}$ (deg, positive nose-up) \\
\hline \hline VORLAX-FEA (uncoupled) & 4.1185 & 0.6547 & -0.6303 & -2.9403 \\
\hline VORLAX-NASTRAN (uncoupled) & 4.1185 & 0.6548 & -0.6341 & -2.9372 \\
\hline VORLAX-FEA (aeroelastic) & 5.3767 & 0.5698 & -0.4919 & -2.6007 \\
\hline VORLAX-NASTRAN (aeroelastic) & 5.3775 & 0.5699 & -0.4948 & -2.5986 \\
\hline
\end{tabular}

Table 2. Aero-Structural Solutions for Design Test Condition, $q_{\infty}=20 \mathbf{p s f}, C_{L}=0.51$

The results show excellent agreement between the VORLAX-FEA and the VORLAX-NASTRAN modeling, establishing confidence in the FEA research framework used in this study. The percent errors between the VORLAX-FEA model and the VORLAX-NASTRAN model are within $<1 \%$ for the deformation values $W_{t i p}, \Theta_{t i p}$, and $\Delta \phi_{y, t i p}$ for both the uncoupled and the coupled aeroelastic solutions.

\section{Comparison Against Wind Tunnel Test Results}

Wind tunnel data results from the UWAL wind tunnel test are analyzed and compared against results from the static aeroelastic VORLAX-FEA model. Some of the wind tunnel data has been presented in previous literature, ${ }^{6,7}$ and the following sections refer to the corresponding raw and processed data from UWAL wind tunnel run test cases. Figure 16 represents the labeling of the VCCTEF flap section/segments, where section 1 represents the inboard flap section and section 5 represents the most outboard flap section. For each of the flap sections, the camber segments are labeled $\mathrm{A}$ for the innermost camber segment, $\mathrm{B}$ for the middle camber segment, and $\mathrm{C}$ for the trailing edge camber segments. Flap deflections are specified in degrees relative to the previous camber segment's deflection. Positive deflections indicate that the flap is deflected downwards.

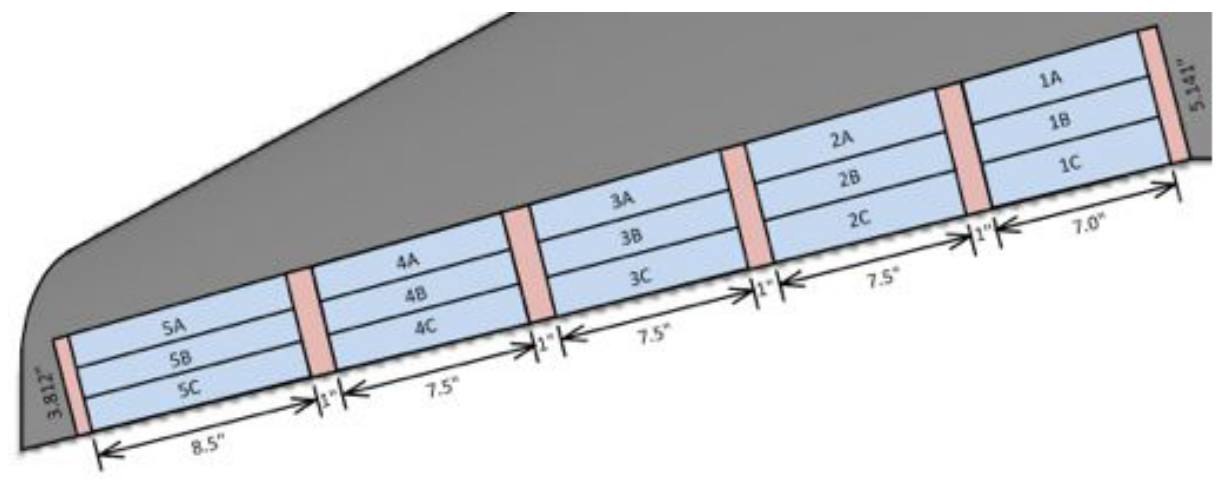

Figure 16. VCCTEF Flap Notation/Label Conventions

The wind tunnel model was tested with a total of 13 VCCTEF configurations ranging from zero to full deflection. These VCCTEF configuration are designated as:

- FLAP0 - baseline zero deflection

- FLAP1 - full deflection for all flap sections

- FLAP2 - varying from a maximum deflection at the inboard and outboard flaps to a minimum deflection at the mid-span flap.

- FLAP3 - varying from a minimum deflection at the inboard and outboard flaps to a maximum deflection at the mid-span flap.

- FLAP4 - varying monotonically from a maximum deflection at the inboard flap to zero deflection at the outboard flap. 
- FLAP5 - varying monotonically from zero deflection at the inboard flap to a maximum deflection at the outboard flap.

- FLAP6 - similar to FLAP4 configuration but with a smaller deflection.

- FLAP7 - varying monotonically from a maximum positive deflection at the inboard flap to a negative deflection at the outboard flap.

- FLAP8 - rigid-body deflection with the two outer camber segments at zero relative deflection.

- FLAP9 - deflection of the trailing edge camber segments.

- FLAP10 - intermediate deflection.

- FLAP11- full negative deflection.

- FLAP12 - FLAP6 configuration plus a gurney flap.

Figure 17 illustrates some of the flap configurations.

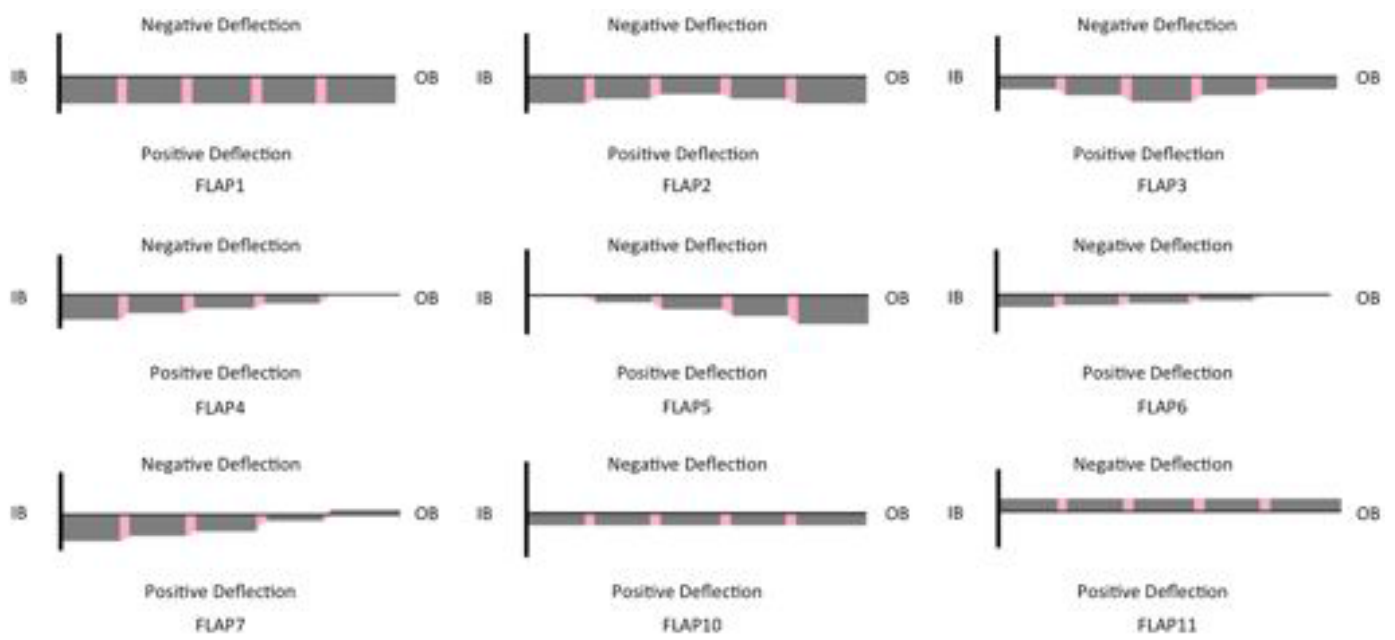

Figure 17. VCCTEF Flap Configurations (Courtesy of UWAL)

A VICON motion tracking system is used in order to measure the aeroelastic deflection of the flexible UWAL model. Details of the VICON tracking set up are included in references. ${ }^{6,7,13}$ The VICON system is also utilized to measure the actual flap deflections of the VCCTEF during a test run. Though the VCCTEF is set to a selected reference flap setting before the wind tunnel is activated, it is observed that the flap deflections deviate from the reference settings with the wind on due to the nature of their construction. VICON tracking data is accepted to be subject to inaccuracy, but lacking other sources of information, measurements from the VICON system for VCCTEF deflections are used. Some of the aeroelastic deflection data is processed and included in this study.

Though a total of 125 useful test runs were conducted by UWAL, only data pertaining to the cases in which the VCCTEF deflections were reported by the VICON tracking system are analyzed in this study. Also, only the cases where the wind tunnel was operating "on condition" at $q_{\infty}=20 \mathrm{psf}$ are examined. Thus, a set of 12 comparison cases are presented in this study corresponding to processed UWAL wind tunnel results for the FLAP0 to FLAP11 configurations. For each comparison case, aerodynamic data from the UWAL wind tunnel test are presented against data generated using the static aeroelastic model. Aeroelastic model results are presented for the VORLAX-FEA model only, which was validated to have very good agreement with the VORLAX-NASTRAN model.

\section{A. FLAP0}

Data corresponding to wind tunnel runs where the VCCTEF is set at zero deflection, or FLAP0, corresponds to runs $18,19,20,21,22,23,24,69,70,71$, and 72 . Table 3 represents VCCTEF flap deflection data for each of the 
sections/segments. The row labeled "Reference" represents the VCCTEF values that are physically set on the model without the wind on. The following rows in Table 3 represent the VCCTEF deflections that are measured by the VICON tracking dots. The VICON measurements indicate a very large spread of data for the VCCTEF deflections (up to $5.4^{\circ}$ of deviation). To reduce any measurement inaccuracies that could be in individual VICON measurements, the mean of the VICON measured VCCTEF deflections is determined and included in Table 3 in the row "Mean".

\begin{tabular}{|c|c|c|c|c|c|c|c|c|c|c|c|c|c|c|c|}
\hline \multirow[b]{2}{*}{ Run } & \multicolumn{15}{|c|}{ VCCTEF Section/Segment Deflection } \\
\hline & $1 \mathrm{~A}$ & 1B & $1 \mathrm{C}$ & $2 \mathrm{~A}$ & $2 \mathrm{~B}$ & $2 \mathrm{C}$ & $3 \mathrm{~A}$ & 3B & $3 \mathrm{C}$ & $4 \mathrm{~A}$ & 4B & $4 \mathrm{C}$ & $5 \mathrm{~A}$ & $5 \mathrm{~B}$ & $5 \mathrm{C}$ \\
\hline Reference & $\mathbf{0 . 0}$ & $\mathbf{0 . 0}$ & $\mathbf{0 . 0}$ & $\mathbf{0 . 0}$ & $\mathbf{0 . 0}$ & $\mathbf{0 . 0}$ & $\mathbf{0 . 0}$ & $\mathbf{0 . 0}$ & $\mathbf{0 . 0}$ & $\mathbf{0 . 0}$ & $\mathbf{0 . 0}$ & $\mathbf{0 . 0}$ & $\mathbf{0 . 0}$ & $\mathbf{0 . 0}$ & $\mathbf{0 . 0}$ \\
\hline 18 & -0.1 & 0.0 & -0.3 & 0.4 & -1.1 & 2.1 & -0.2 & -1.1 & 2.6 & -1.9 & 2.9 & -2.1 & 0.6 & -0.9 & 1.5 \\
\hline 19 & 0.3 & 0.0 & 0.2 & 0.0 & 0.4 & 0.8 & 1.0 & -2.4 & 4.7 & -0.1 & 1.2 & -1.2 & -0.3 & 1.4 & -0.1 \\
\hline 20 & 0.7 & 0.1 & 0.3 & 0.4 & -0.2 & 1.7 & 0.5 & -0.9 & 3.4 & 0.4 & 1.1 & -0.7 & 0.2 & 1.1 & -0.8 \\
\hline 21 & 0.0 & 0.1 & 0.2 & -0.1 & 0.2 & 0.1 & -0.2 & -0.7 & 3.4 & -0.7 & 0.9 & -0.5 & 0.6 & 0.4 & 1.2 \\
\hline 22 & 0.5 & -0.3 & 0.4 & 0.4 & -0.6 & 0.7 & -0.5 & -0.1 & 2.2 & -0.8 & 0.6 & -0.1 & 1.6 & -1.5 & 3.7 \\
\hline 23 & 1.7 & 0.5 & 1.1 & 1.6 & 0.4 & 2.3 & 1.6 & -1.0 & 5.4 & 2.4 & 0.2 & 2.2 & 2.7 & -1.6 & 3.8 \\
\hline 24 & 1.9 & 0.6 & 1.2 & 1.9 & 0.1 & 3.3 & 2.5 & -1.2 & 5.0 & 2.1 & 1.8 & 0.1 & 1.7 & -0.7 & 4.1 \\
\hline 69 & 0.3 & 0.2 & -0.5 & 0.7 & -0.7 & 0.8 & 0.4 & -0.4 & 1.1 & -0.6 & 1.1 & -0.9 & 1.3 & -0.8 & -1.5 \\
\hline 70 & 1.0 & 0.0 & 0.9 & 0.7 & 0.8 & 0.4 & 1.0 & -1.2 & 3.9 & 0.0 & 1.8 & -0.9 & 1.7 & -2.1 & 1.7 \\
\hline 71 & 1.5 & 0.5 & 0.7 & 1.7 & 0.3 & 1.7 & 1.2 & 0.6 & 1.5 & 1.4 & 0.6 & 1.4 & 1.5 & 0.3 & -0.3 \\
\hline 72 & 2.2 & 0.9 & 0.6 & 3.0 & 0.0 & 2.6 & 1.8 & 1.7 & 0.4 & 2.8 & 1.4 & 0.5 & 2.1 & -0.8 & 1.8 \\
\hline Mean & 0.91 & 0.24 & 0.44 & 0.97 & -0.04 & 1.50 & 0.83 & -0.61 & 3.05 & 0.45 & 1.23 & -0.20 & 1.25 & -0.47 & 1.37 \\
\hline
\end{tabular}

Table 3. VCCTEF Deflections and Corresponding Runs for FLAP0 Cases

The static aeroelastic VORLAX-FEA model is run for the flexible model using the reference VCCTEF deflections, the mean VCCTEF deflections, and for the corresponding $\alpha$ and the VICON measured VCCTEF deflections for each of the individual runs. The lift curve and drag polar for the flexible UWAL wind tunnel model are shown in Fig. 18.
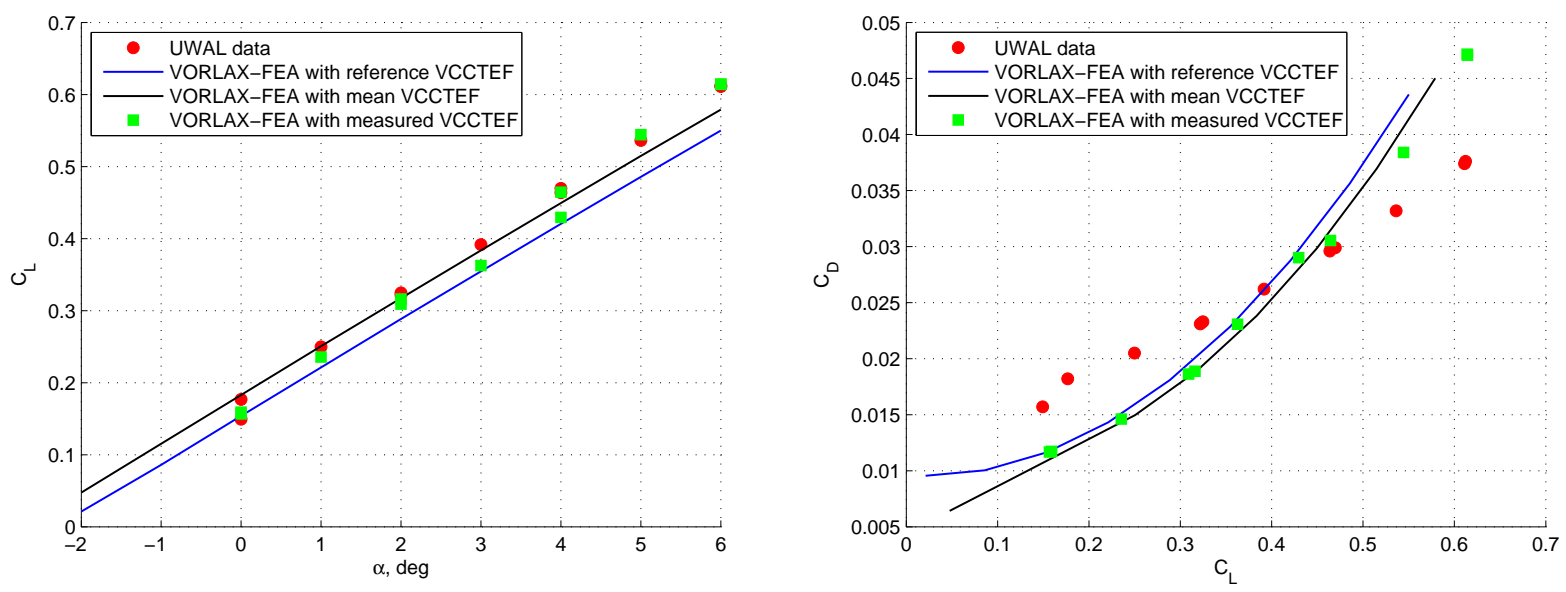

Figure 18. Flexible UWAL Wind Tunnel Model Lift Curve and Drag Polar for FLAP0 Cases

From the Fig. 18, it is seen that the lift curve using the reference set VCCTEF deflection values has a good match in lift curve slope but is offset in $C_{L_{0}}$. Using the mean VCCTEF deflection corrects this issue and the lift curve falls through the UWAL data run points. For completeness, the VORLAX-FEA model results are plotted using the VICON measured VCCTEF deflections. It is clear that a wide spread of data is present.

The values for the lift curve slope and zero angle of attack lift are tabulated in Table 4, and the percent error values are calculated relative to the UWAL data. 


\begin{tabular}{|c|c|c|c|c|}
\hline & $C_{L_{\alpha}}$ & $C_{L_{\alpha}} \%$ Difference & $C_{L_{0}}$ & $C_{L_{0}} \%$ Difference \\
\hline \hline UWAL Data & 4.1290 & 0 & 0.1768 & 0 \\
\hline VORLAX-FEA with reference VCCTEF & 3.8017 & -7.93 & 0.1541 & -12.84 \\
\hline VORLAX-FEA with mean VCCTEF & 3.8106 & -7.71 & 0.1826 & 3.26 \\
\hline
\end{tabular}

Table 4. Flexible UWAL Wind Tunnel Model Lift Curve Comparison, FLAP0 Cases

Good agreement to the UWAL data exists for both the VORLAX-FEA with reference VCCTEF and the VORLAXFEA with mean VCCTEF in terms of $C_{L_{\alpha}}$, approximately $-7.93 \%$ and $-7.71 \%$ respectively, while the VORLAX-FEA with mean VCCTEF measurements is able to predict $C_{L_{0}}$ more accurately.

For the FLAP0 configuration, there are enough VICON measurements such that the wing deflection during the wind tunnel runs can be compared against the static aeroelastic VORLAX-FEA model. Based on data from runs 18, $19,20,23,24,69,70,71$, and 72 , the vertical deflection $W$ of the flexible wind tunnel model that would be experienced at $C_{L}=0.51$ is interpolated and compared against the static deflection determined from the VORLAX-FEA model. The results are shown in Fig. 19.

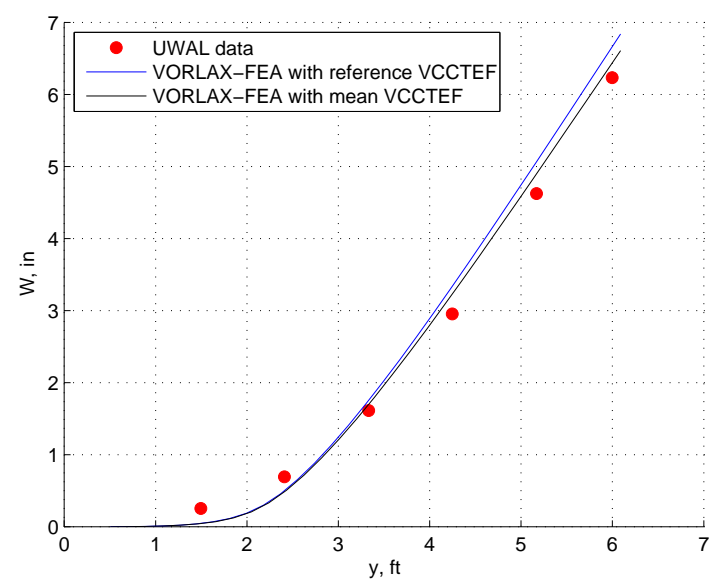

Figure 19. Flexible UWAL Wind Tunnel Model Deflection for FLAP0 Cases

\section{B. FLAP1}

The "flaps full deflection" or FLAP1 runs include runs 25, 26, 27, 28, 29, and 30. Table 5 presents data for the VCCTEF flap deflection values for each of the sections/segments in the same format as was presented in Table 3 for the FLAP0 case comparisons. 


\begin{tabular}{|c|c|c|c|c|c|c|c|c|c|c|c|c|c|c|c|}
\hline & \multicolumn{15}{|c|}{ VCCTEF Section/Segment Deflection } \\
\hline Run & $1 \mathrm{~A}$ & $1 \mathrm{~B}$ & $1 \mathrm{C}$ & $2 \mathrm{~A}$ & 2B & $2 \mathrm{C}$ & $3 \mathrm{~A}$ & 3B & $3 \mathrm{C}$ & $4 \mathrm{~A}$ & 4B & $4 \mathrm{C}$ & $5 \mathrm{~A}$ & $5 \mathrm{~B}$ & $5 \mathrm{C}$ \\
\hline Reference & 5.0 & 4.0 & 9.0 & 6.0 & 7.0 & 9.0 & 9.0 & 7.0 & 10.0 & 7.0 & 8.0 & 10.0 & 6.0 & 9.0 & 9.0 \\
\hline 25 & 1.0 & 2.5 & 10.2 & 4.4 & 3.5 & 15.6 & 2.9 & 7.6 & 13.8 & 3.2 & 6.5 & 15.6 & 2.9 & 2.3 & 10.9 \\
\hline 26 & 2.3 & 1.9 & 11.0 & 5.4 & 3.8 & 15.9 & 4.9 & 6.1 & 15.3 & 5.7 & 4.4 & 18.8 & 1.2 & 4.0 & 8.7 \\
\hline 27 & 1.2 & 2.6 & 9.6 & 4.1 & 3.6 & 14.9 & 3.3 & 7.1 & 13.7 & 3.2 & 5.2 & 15.8 & 1.4 & 4.9 & 8.5 \\
\hline 28 & 2.7 & 2.0 & 10.7 & 6.0 & 3.1 & 16.4 & 5.3 & 5.1 & 16.3 & 5.6 & 5.2 & 16.9 & 1.8 & 2.6 & 10.1 \\
\hline 29 & 2.8 & 2.1 & 10.5 & 5.4 & 3.8 & 15.3 & 4.4 & 7.1 & 13.7 & 5.0 & 5.2 & 16.7 & 3.2 & 0.3 & 12.7 \\
\hline 30 & 0.7 & 2.7 & 9.9 & 3.6 & 4.4 & 13.9 & 4.0 & 5.7 & 5.7 & 3.7 & 4.3 & 16.9 & 0.7 & 5.7 & 7.2 \\
\hline Mean & 1.78 & 2.30 & 10.32 & 4.82 & 3.70 & 15.33 & 4.13 & 6.45 & 14.67 & 4.40 & 5.13 & 16.78 & 1.87 & 3.30 & 9.68 \\
\hline
\end{tabular}

Table 5. VCCTEF Deflections and Corresponding Runs for FLAP1 Cases

Flexible UWAL wind tunnel model lift curves and drag polars are generated using the VORLAX-FEA aeroelastic model for the reference VCCTEF deflections and the mean VCCTEF deflections. The VORLAX-FEA model is also run at the individual $\alpha$ and VICON measured VCCTEF deflections for each of the individual runs. The results are shown in Fig. 20.
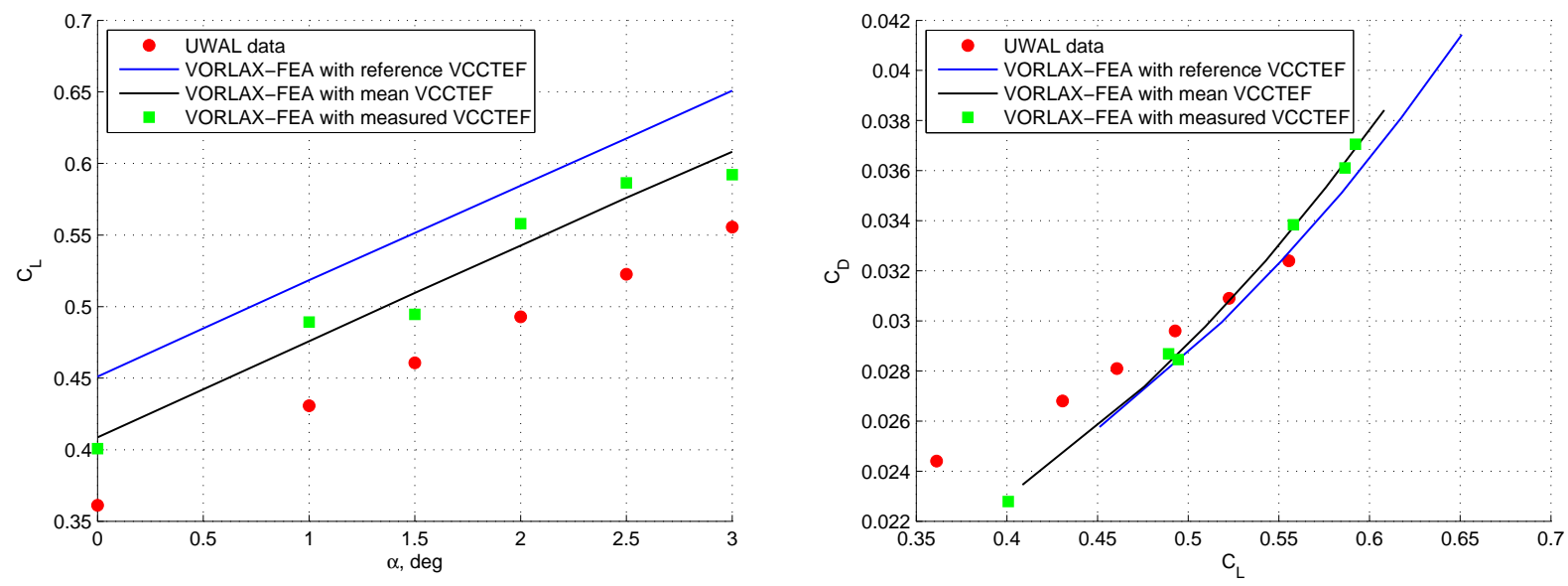

Figure 20. Flexible UWAL Wind Tunnel Model Lift Curve and Drag Polar for FLAP1 Cases

The values for the lift curve slope and zero angle of attack lift are tabulated in Table 6 and the percent error values are calculated relative to the UWAL data.

\begin{tabular}{|c|c|c|c|c|}
\hline & $C_{L_{\alpha}}$ & $C_{L_{\alpha}} \%$ Difference & $C_{L_{0}}$ & $C_{L_{0}} \%$ Difference \\
\hline \hline UWAL Data & 3.6786 & 0 & 0.3636 & 0 \\
\hline VORLAX-FEA with reference VCCTEF & 3.8085 & 3.53 & 0.4515 & 24.19 \\
\hline VORLAX-FEA with mean VCCTEF & 3.8163 & 3.74 & 0.4091 & 12.52 \\
\hline
\end{tabular}

Table 6. Flexible UWAL Wind Tunnel Model Lift Curve Comparison, FLAP1 Cases

\section{FLAP2}

The FLAP2 configuration refers to the flap configuration where the VCCTEF is at maximum deflection at the inboard and outboard sections while at a minimum deflection at the mid-span. The set of runs corresponding to this configuration are runs 32, 33, 34, 35, and 36. The VCCTEF flap deflection data for each of the sections/segments are presented in Table 7. 


\begin{tabular}{|c|c|c|c|c|c|c|c|c|c|c|c|c|c|c|c|}
\hline \multirow[b]{2}{*}{ Run } & \multicolumn{15}{|c|}{ VCCTEF Section/Segment Deflection } \\
\hline & $1 \mathrm{~A}$ & 1B & $1 \mathrm{C}$ & $2 \mathrm{~A}$ & $2 \mathrm{~B}$ & $2 \mathrm{C}$ & $3 \mathrm{~A}$ & $3 \mathrm{~B}$ & $3 \mathrm{C}$ & $4 \mathrm{~A}$ & 4B & $4 \mathrm{C}$ & $5 \mathrm{~A}$ & $5 \mathrm{~B}$ & $5 \mathrm{C}$ \\
\hline Reference & 3.0 & 2.0 & 10.0 & 4.0 & 5.0 & 4.0 & 5.0 & 3.0 & 4.0 & 4.0 & 6.0 & 5.0 & 5.0 & 10.0 & $\mathbf{1 0 . 0}$ \\
\hline 32 & 0.2 & 2.6 & 8.2 & 1.5 & 1.4 & 9.2 & 0.3 & 2.8 & 5.4 & -0.3 & 4.6 & 6.3 & -0.1 & 4.3 & 807 \\
\hline 33 & 0.2 & 2.5 & 8.9 & 0.9 & 2.7 & 6.9 & 2.1 & 1.2 & 6.6 & 0.6 & 3.2 & 7.7 & -1.2 & 6.9 & 4.6 \\
\hline 34 & 1.4 & 2.6 & 9.0 & 3.0 & 1.6 & 9.7 & 3.6 & 1.2 & 7.8 & 5.4 & -0.3 & 12.2 & 1.7 & 2.5 & 10.4 \\
\hline 35 & 0.8 & 2.4 & 9.5 & 2.4 & 1.9 & 8.3 & 1.7 & 2.6 & 6.3 & 4.5 & 0.1 & 11.0 & 0.0 & 4.3 & 8.8 \\
\hline 36 & 2.0 & 2.4 & 9.8 & 2.8 & 2.9 & 8.5 & 4.3 & 0.8 & 8.6 & 9.8 & -4.4 & 16.4 & 0.5 & 4.1 & 9.0 \\
\hline Mean & 0.92 & 2.50 & 9.08 & 2.12 & 2.10 & 8.52 & 2.40 & 1.72 & 6.94 & 4.00 & 0.64 & 10.72 & 0.18 & 4.42 & 8.3 \\
\hline
\end{tabular}

Table 7. VCCTEF Deflections and Corresponding Runs for FLAP2 Cases

Flexible UWAL wind tunnel model lift curves and drag polars are generated using the VORLAX-FEA aeroelastic model for the reference VCCTEF deflections and the mean VCCTEF deflections. The data the corresponding to the measured VCCTEF deflections for each of the individual runs is also plotted to show the spread of data. The results are shown in Fig. 21.
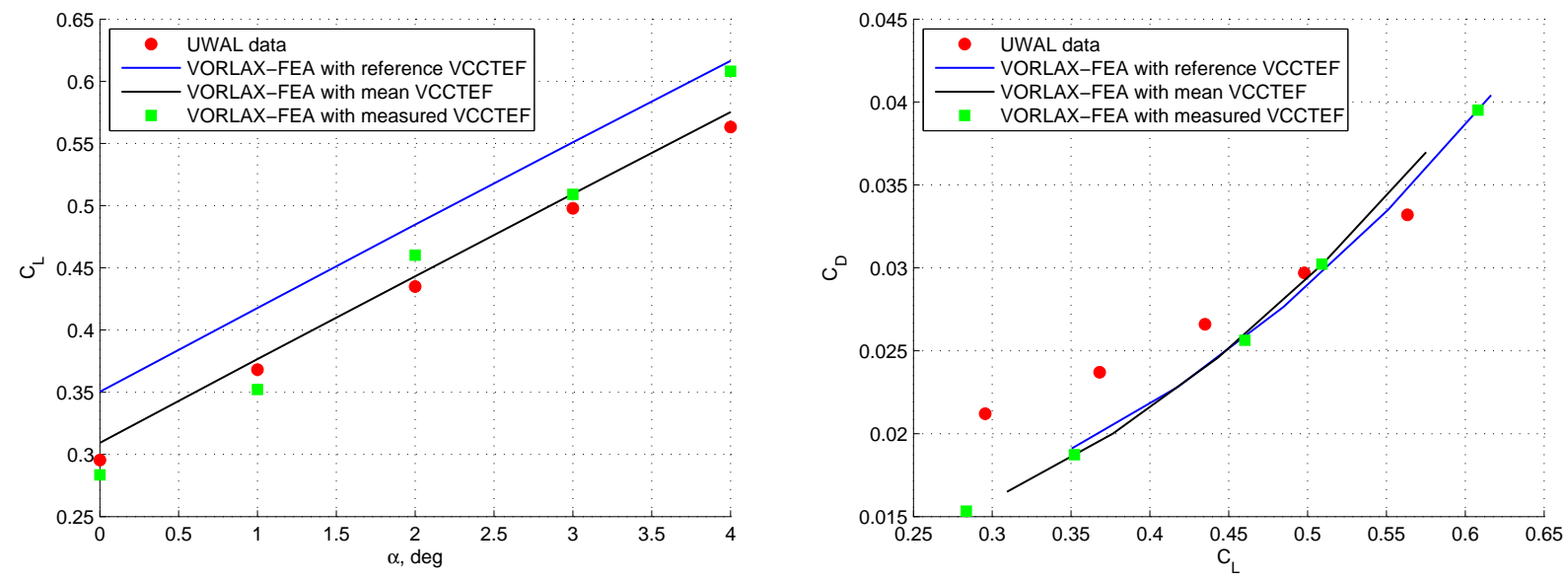

Figure 21. Flexible UWAL Wind Tunnel Model Lift Curve and Drag Polar for FLAP2 Cases

Table 8 shows the values for the lift curve slope and zero angle of attack lift for the VORLAX-FEA model, as well as the the percent error values calculated relative to the UWAL data.

\begin{tabular}{|c|c|c|c|c|}
\hline & $C_{L_{\alpha}}$ & $C_{L_{\alpha}} \%$ Difference & $C_{L_{0}}$ & $C_{L_{0}} \%$ Difference \\
\hline \hline UWAL Data & 3.8125 & 0 & 0.2989 & 0 \\
\hline VORLAX-FEA with reference VCCTEF & 3.8127 & 0.01 & 0.3510 & 17.44 \\
\hline VORLAX-FEA with mean VCCTEF & 3.8090 & -0.09 & 0.3098 & 3.66 \\
\hline
\end{tabular}

Table 8. Flexible UWAL Wind Tunnel Model Lift Curve Comparison, FLAP2 Cases

\section{FLAP3}

The next case examined in this study corresponds to the FLAP3 configuration, which refers to the flap configuration where the VCCTEF is at minimum deflection at the inboard and outboard sections while at maximum deflection at the mid-span. Runs 37, 38, 39, 40, and 41 correspond to data at this case. The VCCTEF flap deflection data for each of the sections/segments are presented in Table 9. 


\begin{tabular}{|c|c|c|c|c|c|c|c|c|c|c|c|c|c|c|c|}
\hline & \multicolumn{15}{|c|}{ VCCTEF Section/Segment Deflection } \\
\hline Run & $1 \mathrm{~A}$ & $1 \mathrm{~B}$ & $1 \mathrm{C}$ & $2 \mathrm{~A}$ & 2B & $2 \mathrm{C}$ & $3 \mathrm{~A}$ & $3 \mathrm{~B}$ & $3 \mathrm{C}$ & $4 \mathrm{~A}$ & $4 \mathrm{~B}$ & $4 \mathrm{C}$ & $5 \mathrm{~A}$ & $5 \mathrm{~B}$ & $5 \mathrm{C}$ \\
\hline Reference & 2.0 & 3.0 & 5.0 & 5.0 & 6.0 & 5.0 & 6.0 & 6.0 & 11.0 & 5.0 & 6.0 & 5.0 & 4.0 & 6.0 & 5.0 \\
\hline 37 & 0.6 & 3.0 & 4.1 & 2.5 & 2.7 & 9.9 & 2.5 & 5.4 & 13.8 & 3.4 & 3.6 & 9.6 & 1.2 & 0.8 & 6.2 \\
\hline 38 & 1.0 & 2.8 & 1.0 & 2.8 & 1.7 & 11.1 & 2.4 & 5.2 & 14.6 & 3.2 & 2.6 & 9.7 & 0.6 & 1.8 & 3.1 \\
\hline 39 & 0.9 & 2.6 & 4.7 & 2.2 & 2.8 & 9.8 & 3.5 & 4.1 & 14.9 & 5.0 & 1.0 & 11.9 & 1.8 & -0.4 & 7.9 \\
\hline 40 & 1.5 & 2.4 & 5.3 & 2.8 & 3.1 & 9.5 & 2.4 & 5.6 & 14.3 & 7.3 & 0.0 & 13.3 & 1.1 & 0.9 & 5.6 \\
\hline 41 & 2.1 & 2.2 & 5.7 & 2.9 & 3.3 & 9.7 & 4.3 & 4.3 & 15.6 & 11.2 & -4.4 & 17.4 & 1.3 & 3.3 & 2.6 \\
\hline Mean & 1.22 & 2.6 & 4.16 & 2.64 & 2.72 & 10.0 & 3.02 & 4.92 & 14.64 & 6.02 & 0.56 & 12.38 & 1.2 & 1.28 & 5.08 \\
\hline
\end{tabular}

Table 9. VCCTEF Deflections and Corresponding Runs for FLAP3 Cases

Flexible UWAL wind tunnel model lift curves and drag polars are generated using the VORLAX-FEA aeroelastic model for the reference VCCTEF deflections and the mean VCCTEF deflections. Individual data points at corresponding $\alpha$ and the VICON measured VCCTEF deflections are shown for the individual runs. The results are shown in Fig. 22.
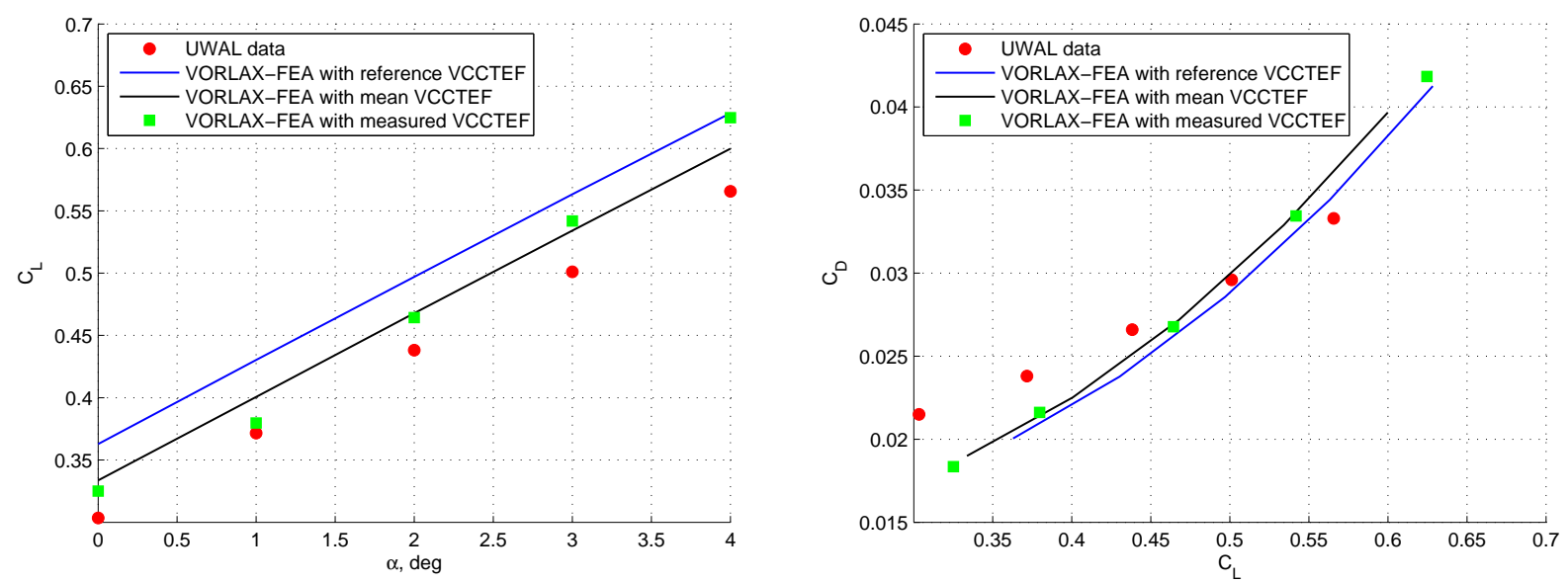

Figure 22. Flexible UWAL Wind Tunnel Model Lift Curve and Drag Polar for FLAP3 Cases

The values for the lift curve slope and zero angle of attack lift are tabulated in Table 10 along with the percent error values calculated relative to the UWAL data.

\begin{tabular}{|c|c|c|c|c|}
\hline & $C_{L_{\alpha}}$ & $C_{L_{\alpha}} \%$ Difference & $C_{L_{0}}$ & $C_{L_{0}} \%$ Difference \\
\hline \hline UWAL Data & 3.7477 & 0 & 0.3052 & 0 \\
\hline VORLAX-FEA with reference VCCTEF & 3.8057 & 1.55 & 0.3635 & 19.12 \\
\hline VORLAX-FEA with mean VCCTEF & 3.8160 & 1.82 & 0.3340 & 9.45 \\
\hline
\end{tabular}

Table 10. Flexible UWAL Wind Tunnel Model Lift Curve Comparison, FLAP3 Cases

\section{E. FLAP4}

The FLAP4 configuration refers to the flap configuration where the VCCTEF deflection is varied monotonically from a maximum deflection at the inboard to zero deflection at the outboard sections. Runs 42, 43, 44, 45, and 46 correspond to data runs at FLAP4. The VCCTEF flap deflection data for each of the sections/segments are presented in Table 11. 


\begin{tabular}{|c|c|c|c|c|c|c|c|c|c|c|c|c|c|c|c|}
\hline & \multicolumn{15}{|c|}{ VCCTEF Section/Segment Deflection } \\
\hline Run & $1 \mathrm{~A}$ & $1 \mathrm{~B}$ & $1 \mathrm{C}$ & $2 \mathrm{~A}$ & 2B & $2 \mathrm{C}$ & $3 \mathrm{~A}$ & 3B & $3 \mathrm{C}$ & $4 \mathrm{~A}$ & 4B & $4 \mathrm{C}$ & $5 \mathrm{~A}$ & $5 \mathrm{~B}$ & $5 \mathrm{C}$ \\
\hline Reference & 2.0 & 5.0 & 9.0 & 3.0 & 6.0 & 3.0 & 4.0 & 1.0 & 6.0 & 2.0 & 5.0 & 2.0 & $\mathbf{0 . 0}$ & 0.0 & $\mathbf{0 . 0}$ \\
\hline 42 & -0.9 & 4.2 & 8.6 & 1.1 & 3.1 & 6.6 & 1.5 & 0.2 & 9.2 & -0.5 & 3.3 & 1.9 & 1.0 & -0.2 & 0.5 \\
\hline 43 & -0.1 & 4.1 & 8.3 & 2.3 & 1.9 & 7.3 & 1.6 & 1.0 & 8.2 & 0.3 & 3.5 & 3.6 & 1.6 & -0.8 & 0.8 \\
\hline 44 & 0.3 & 4.3 & 8.1 & 2.0 & 3.4 & 6.2 & 1.8 & 1.6 & 7.6 & 3.6 & -0.4 & 7.8 & 1.3 & 0.4 & 0.4 \\
\hline 45 & 0.6 & 4.4 & 8.3 & 2.0 & 3.7 & 6.0 & 3.5 & -0.5 & 10.4 & 5.9 & -1.4 & 8.4 & 2.2 & -0.7 & 1.3 \\
\hline 46 & 0.9 & 4.1 & 8.7 & 2.6 & 2.4 & 8.0 & 2.0 & 2.5 & 7.8 & 8.4 & -4.2 & 11.2 & 2.2 & -1.5 & 3.6 \\
\hline Mean & 0.16 & 4.22 & 8.40 & 2.00 & 2.90 & 6.82 & 2.08 & 0.96 & 8.64 & 3.54 & 0.16 & 6.58 & 1.66 & -0.56 & 1.32 \\
\hline
\end{tabular}

Table 11. VCCTEF Deflections and Corresponding Runs for FLAP4 Cases

The VORLAX-FEA aeroelastic model is used to generate lift curve and drag polar data for the model with reference VCCTEF deflections and mean VCCTEF deflections. Individual points are plotted for the VICON measured VCCTEF deflections. The results are shown in Fig. 23.
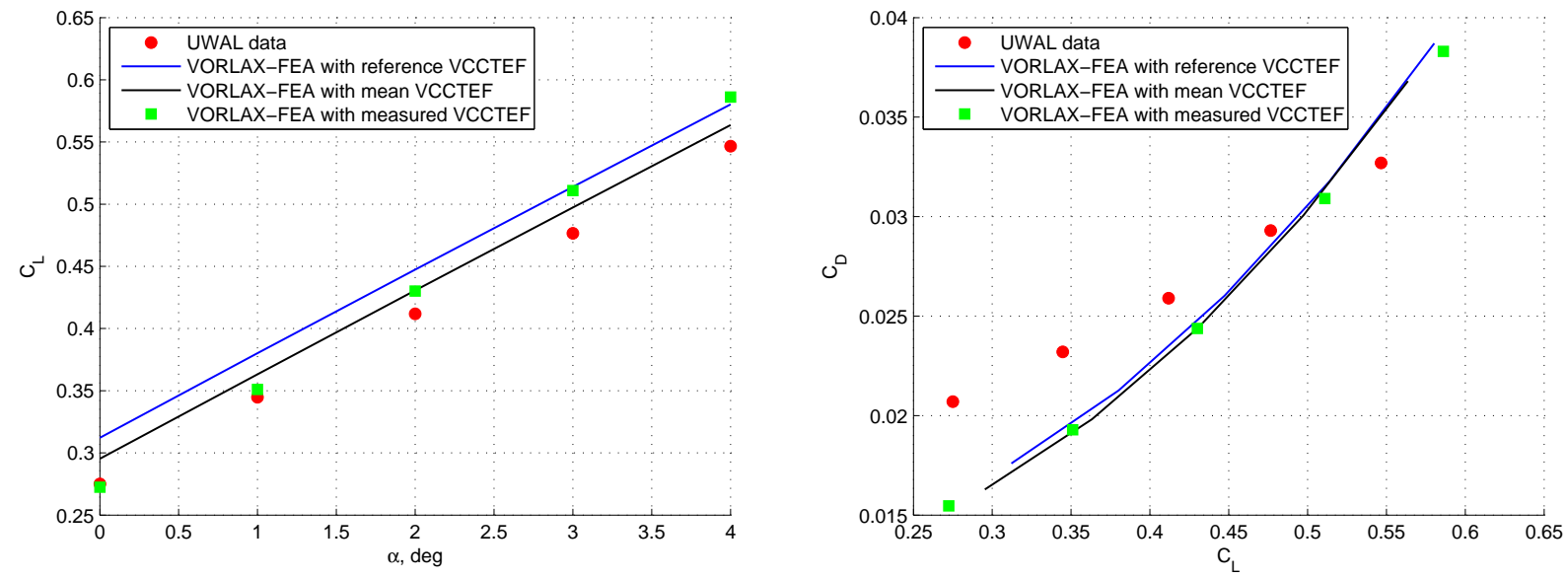

Figure 23. Flexible UWAL Wind Tunnel Model Lift Curve and Drag Polar for FLAP4 Cases

Table 12 shows the lift curve slope parameters generated by the VORLAX-FEA model and the corresponding percent error values relative to the UWAL data.

\begin{tabular}{|c|c|c|c|c|}
\hline & $C_{L_{\alpha}}$ & $C_{L_{\alpha}} \%$ Difference & $C_{L_{0}}$ & $C_{L_{0}} \%$ Difference \\
\hline \hline UWAL Data & 3.8680 & 0 & 0.2759 & 0 \\
\hline VORLAX-FEA with reference VCCTEF & 3.8034 & -1.67 & 0.3090 & 11.99 \\
\hline VORLAX-FEA with mean VCCTEF & 3.8076 & -1.56 & 0.2921 & 5.87 \\
\hline
\end{tabular}

Table 12. Flexible UWAL Wind Tunnel Model Lift Curve Comparison, FLAP4 Cases

\section{F. FLAP5}

The FLAP5 configuration refers to the flap configuration where the VCCTEF is varied monotonically from zero deflection at the inboard flap to a maximum deflection at the outboard sections. The FLAP5 runs correspond to runs 48, 49, 50, 51, 52, and 53. VCCTEF flap deflection data for these runs are presented in Table 13. 


\begin{tabular}{|c|c|c|c|c|c|c|c|c|c|c|c|c|c|c|c|}
\hline \multirow[b]{2}{*}{ Run } & \multicolumn{15}{|c|}{ VCCTEF Section/Segment Deflection } \\
\hline & $1 \mathrm{~A}$ & $1 \mathrm{~B}$ & $1 \mathrm{C}$ & $2 \mathrm{~A}$ & 2B & $2 \mathrm{C}$ & $3 \mathrm{~A}$ & 3B & $3 \mathrm{C}$ & $4 \mathrm{~A}$ & 4B & $4 \mathrm{C}$ & $5 \mathrm{~A}$ & $5 \mathrm{~B}$ & $5 \mathrm{C}$ \\
\hline Reference & $\mathbf{0 . 0}$ & $\mathbf{0 . 0}$ & $\mathbf{0 . 0}$ & 2.0 & 5.0 & 1.0 & 4.0 & 2.0 & 7.0 & 3.0 & 5.0 & 6.0 & 3.0 & 8.0 & 9.0 \\
\hline 48 & 0.3 & 1.6 & -1.8 & 2.0 & 2.2 & 4.2 & 3.3 & 0.4 & 10.4 & 3.2 & 2.2 & 11.6 & 0.5 & 5.4 & 8.6 \\
\hline 49 & 0.8 & 1.9 & -1.6 & 2.2 & 2.3 & 4.7 & 2.9 & 1.4 & 10.1 & 3.3 & 3.1 & 11.4 & 0.6 & 3.9 & 9.7 \\
\hline 50 & 1.2 & 1.6 & -0.8 & 3.4 & 0.4 & 7.3 & 3.7 & 1.1 & 10.1 & 5.7 & 0.8 & 13.5 & 1.5 & 2.6 & 12.4 \\
\hline 51 & 1.6 & 1.5 & -0.2 & 4.1 & 0.5 & 7.1 & 3.1 & 2.2 & 9.9 & 7.2 & 0.1 & 14.6 & 1.6 & 4.7 & 8.6 \\
\hline 52 & 0.3 & 1.0 & -1.0 & 1.4 & 1.8 & 3.9 & 1.8 & 0.7 & 9.4 & 4.8 & 0.8 & 11.8 & 1.7 & 4.5 & 9.8 \\
\hline 53 & 2.2 & 1.7 & 0.4 & 3.5 & 2.4 & 5.8 & 5.0 & -0.4 & 12.8 & 6.3 & 1.0 & 13.9 & 1.6 & 4.2 & 10.1 \\
\hline Mean & 1.07 & 1.55 & -0.83 & 2.77 & 1.60 & 5.50 & 3.30 & 0.90 & 10.45 & 5.08 & 1.33 & 128 & 1.25 & 4.22 & 9.87 \\
\hline
\end{tabular}

Table 13. VCCTEF Deflections and Corresponding Runs for FLAP5 Cases

Lift curve results from the VORLAX-FEA model are shown in Fig. 24.
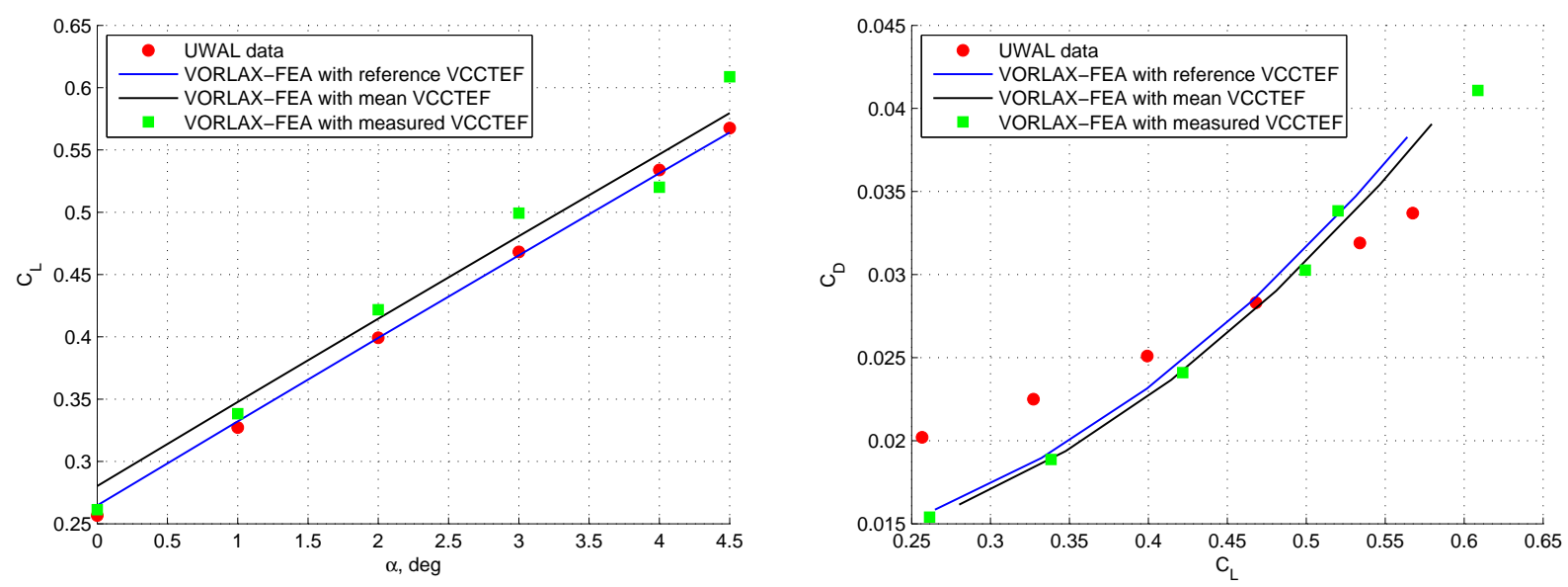

Figure 24. Flexible UWAL Wind Tunnel Model Lift Curve and Drag Polar for FLAP5 Cases

The parameters for the lift curves are tabulated in Table 14, and percent error values relative to the UWAL data are presented.

\begin{tabular}{|c|c|c|c|c|}
\hline & $C_{L_{\alpha}}$ & $C_{L_{\alpha}} \%$ Difference & $C_{L_{0}}$ & $C_{L_{0}} \%$ Difference \\
\hline \hline UWAL Data & 3.9585 & 0 & 0.2585 & 0 \\
\hline VORLAX-FEA with reference VCCTEF & 3.8494 & -3.77 & 0.2655 & 2.70 \\
\hline VORLAX-FEA with mean VCCTEF & 3.8080 & -3.80 & 0.2809 & 8.69 \\
\hline
\end{tabular}

Table 14. Flexible UWAL Wind Tunnel Model Lift Curve Comparison, FLAP5 Cases

\section{G. FLAP6}

Runs 55, 56, 57, 58, 59, 61, and 62 correspond to data taken with the FLAP6 configuration. This VCCTEF configuration varies monotonically from a small deflection at the inboard section to zero deflection at the outboard section. VCCTEF flap deflection data is presented in Table 15. 


\begin{tabular}{|c|c|c|c|c|c|c|c|c|c|c|c|c|c|c|c|}
\hline \multirow[b]{2}{*}{ Run } & \multicolumn{15}{|c|}{ VCCTEF Section/Segment Deflection } \\
\hline & $1 \mathrm{~A}$ & 1B & $1 \mathrm{C}$ & $2 \mathrm{~A}$ & 2B & $2 \mathrm{C}$ & $3 \mathrm{~A}$ & $3 \mathrm{~B}$ & $3 \mathrm{C}$ & $4 \mathrm{~A}$ & 4B & $4 \mathrm{C}$ & $5 \mathrm{~A}$ & $5 \mathrm{~B}$ & $5 \mathrm{C}$ \\
\hline Reference & 1.0 & 1.0 & 1.0 & $\mathbf{0 . 0}$ & 2.0 & $\mathbf{0 . 0}$ & $\mathbf{0 . 0}$ & $\mathbf{0 . 0}$ & $\mathbf{0 . 0}$ & 2.0 & 0.0 & $\mathbf{0 . 0}$ & 0.5 & 0.0 & $\mathbf{0 . 0}$ \\
\hline 55 & 0.2 & 0.5 & 1.4 & -0.1 & 0.7 & 2.5 & -0.1 & 0.8 & 1.8 & 0.0 & 0.5 & 2.3 & 0.5 & -0.3 & 0.1 \\
\hline 56 & 0.8 & 0.6 & 1.3 & 0.6 & 0.9 & 2.8 & 0.4 & 0.0 & 4.6 & -0.4 & 2.2 & 0.5 & -0.1 & 2.1 & -2.6 \\
\hline 57 & 1.1 & 0.5 & 2.0 & 1.0 & 1.5 & 2.3 & 0.2 & 1.6 & 2.6 & 1.3 & 0.4 & 2.4 & 2.6 & -3.0 & 3.5 \\
\hline 58 & 1.3 & 0.5 & 2.6 & 0.9 & 1.4 & 2.4 & 0.1 & 2.4 & 2.0 & 1.8 & 0.4 & 3.4 & 1.7 & -0.3 & -0.8 \\
\hline 59 & 1.6 & 0.5 & 2.6 & 1.0 & 1.5 & 2.6 & 0.7 & 1.7 & 3.5 & 1.9 & 1.5 & 2.0 & 1.9 & 2.3 & -3.3 \\
\hline 61 & 2.0 & 0.6 & 3.1 & 1.1 & 2.6 & 2.1 & 1.4 & 2.0 & 3.0 & 2.6 & 1.6 & 2.5 & 1.3 & 3.0 & -3.6 \\
\hline 62 & 2.2 & 0.5 & 3.4 & 1.7 & 1.9 & 3.2 & 1.6 & 0.8 & 5.4 & 2.0 & 1.8 & 3.7 & 1.9 & 1.4 & -1.0 \\
\hline Mean & 1.31 & 0.53 & 2.34 & 0.89 & 1.5 & 2.56 & 0.61 & 1.33 & 3.27 & 1.31 & 1.20 & 2.40 & 1.40 & 0.74 & -1.10 \\
\hline
\end{tabular}

Table 15. VCCTEF Deflections and Corresponding Runs for FLAP6 Cases

Flexible UWAL wind tunnel model lift curves and drag polars results are shown in Fig. 25.
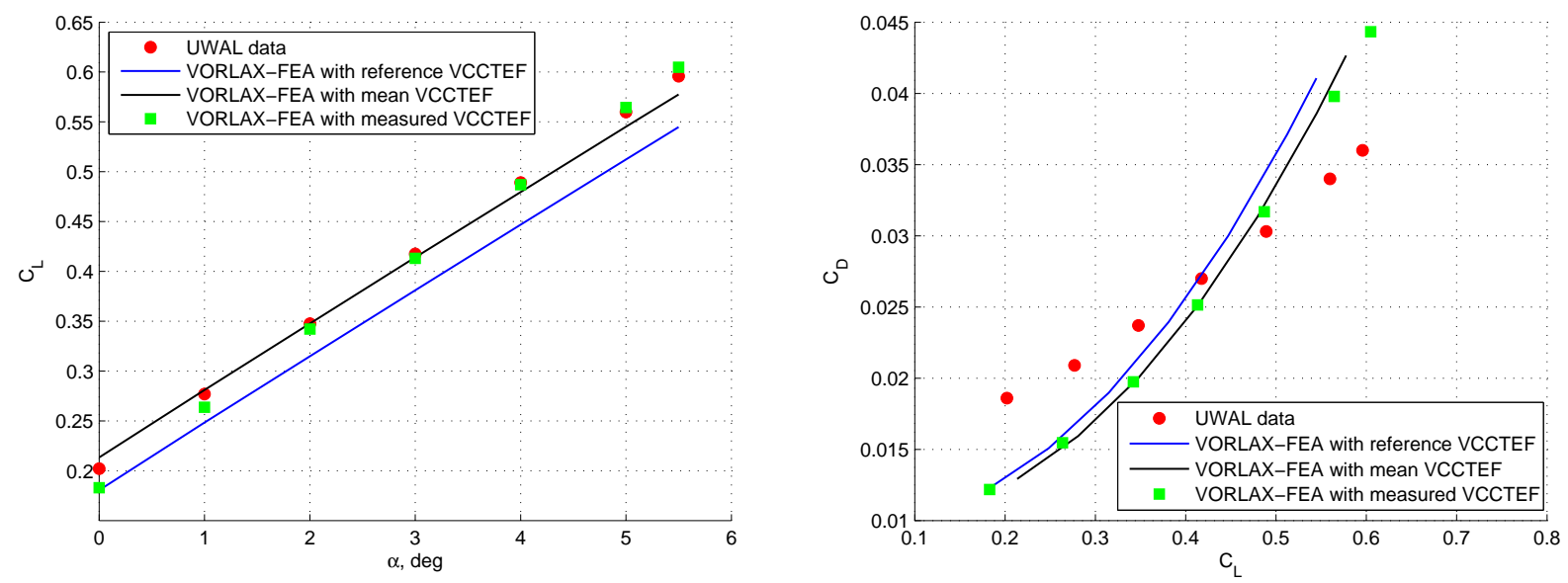

Figure 25. Flexible UWAL Wind Tunnel Model Lift Curve and Drag Polar for FLAP6 Cases

Table 16 summarizes the lift curve slope, zero angle of attack lift, and their percent error values relative to the UWAL data.

\begin{tabular}{|c|c|c|c|c|}
\hline & $C_{L_{\alpha}}$ & $C_{L_{\alpha}} \%$ Difference & $C_{L_{0}}$ & $C_{L_{0}} \%$ Difference \\
\hline \hline UWAL Data & 4.0833 & 0 & 0.2040 & 0 \\
\hline VORLAX-FEA with reference VCCTEF & 3.7908 & -7.16 & 0.1818 & -10.89 \\
\hline VORLAX-FEA with mean VCCTEF & 3.7907 & -7.17 & 0.2146 & 5.21 \\
\hline
\end{tabular}

Table 16. Flexible UWAL Wind Tunnel Model Lift Curve Comparison, FLAP6 Cases

\section{H. FLAP7}

The FLAP7 configuration varies monotonically from a maximum positive deflection at the inboard flap to a negative deflection at the outboard flap. Runs number $63,64,65,66,67$, and 68 correspond to data collected with the FLAP7 configuration. Table 17 summarizes the flap deflections for the sequence of runs. 


\begin{tabular}{|c|c|c|c|c|c|c|c|c|c|c|c|c|c|c|c|}
\hline \multirow[b]{2}{*}{ Run } & \multicolumn{15}{|c|}{ VCCTEF Section/Segment Deflection } \\
\hline & $1 \mathrm{~A}$ & $1 \mathrm{~B}$ & $1 \mathrm{C}$ & $2 \mathrm{~A}$ & 2B & $2 \mathrm{C}$ & $3 \mathrm{~A}$ & 3B & $3 \mathrm{C}$ & $4 \mathrm{~A}$ & 4B & $4 \mathrm{C}$ & $5 \mathrm{~A}$ & $5 \mathrm{~B}$ & $5 \mathrm{C}$ \\
\hline Reference & 3.0 & 3.0 & 4.0 & 2.0 & 3.0 & 2.0 & $\mathbf{0 . 0}$ & $\mathbf{0 . 0}$ & 3.0 & $\mathbf{0 . 0}$ & 0.0 & $\mathbf{0 . 0}$ & $\mathbf{0 . 0}$ & 0.0 & -2.0 \\
\hline 63 & 0.3 & 1.7 & 4.7 & 0.8 & 1.4 & 5.0 & 0.9 & -0.2 & 4.5 & -0.5 & 1.4 & 0.0 & 0.8 & -1.1 & -1.4 \\
\hline 64 & 0.3 & 2.3 & 4.5 & 1.4 & 1.6 & 5.1 & 0.6 & 0.7 & 3.2 & 0.8 & 0.8 & 1.2 & 2.2 & -2.9 & -0.6 \\
\hline 65 & 0.7 & 2.0 & 4.8 & 0.8 & 2.7 & 3.9 & 1.1 & 1.0 & 3.1 & 0.8 & 0.9 & 1.0 & -0.1 & 2.6 & -4.2 \\
\hline 66 & 1.2 & 1.4 & 5.9 & 1.2 & 1.7 & 5.2 & 1.0 & 1.8 & 3.2 & 1.7 & 0.5 & 2.8 & 2.0 & -1.7 & -0.2 \\
\hline 67 & 1.6 & 1.1 & 6.5 & 2.0 & 1.5 & 5.1 & 1.5 & 1.1 & 4.2 & 1.4 & 1.9 & 1.2 & 2.9 & -2.5 & 1.3 \\
\hline 68 & 2.0 & 1.1 & 7.2 & 1.9 & 2.4 & 5.1 & 1.5 & 2.5 & 2.8 & 2.4 & 2.1 & 1.3 & 2.0 & 0.0 & -1.7 \\
\hline Mean & 1.02 & 1.60 & 5.60 & 1.35 & 1.88 & 4.90 & 1.10 & 1.15 & 3.5 & 1.10 & 1.27 & 1.25 & 1.63 & -0.93 & -1.13 \\
\hline
\end{tabular}

Table 17. VCCTEF Deflections and Corresponding Runs for FLAP7 Cases

Figure 26 represents the plots of the lift curve and drag polar generated by the VORLAX-FEA aeroelastic model and the data from the UWAL run.
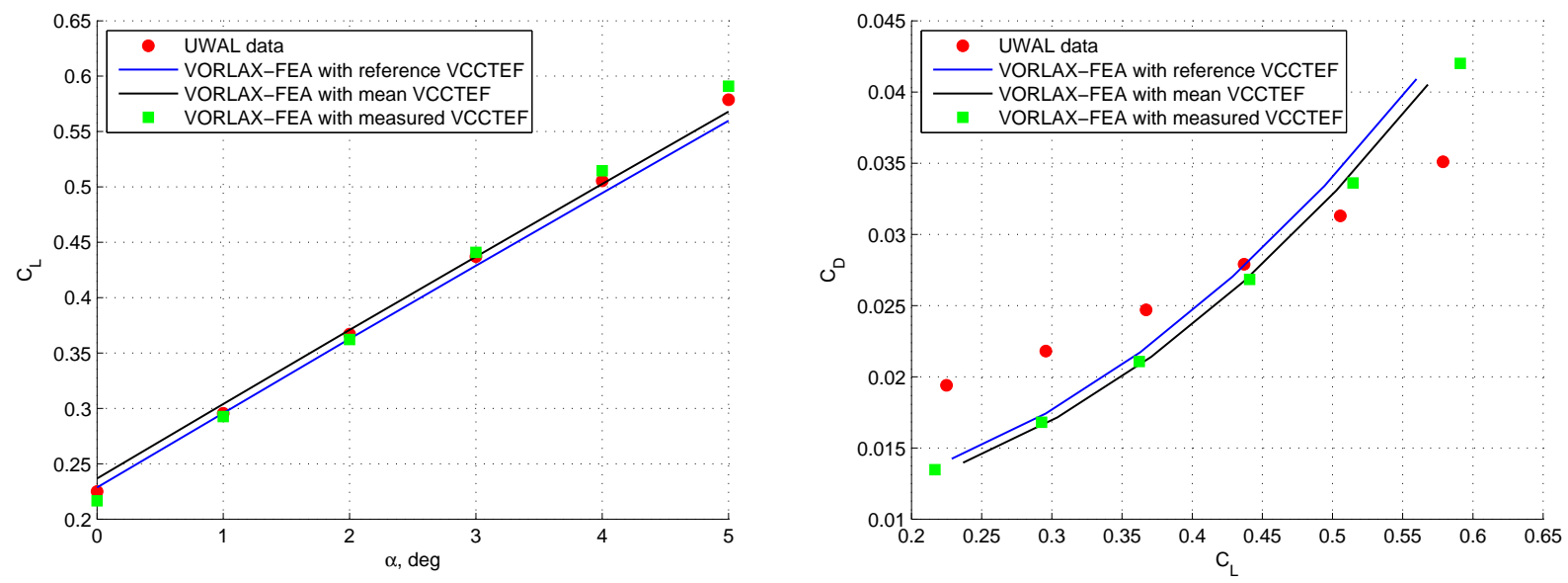

Figure 26. Flexible UWAL Wind Tunnel Model Lift Curve and Drag Polar for FLAP7 Cases

Table 18 summarizes the lift curve parameters from the VORLAX-FEA model and their percent error values relative to the UWAL data.

\begin{tabular}{|c|c|c|c|c|}
\hline & $C_{L_{\alpha}}$ & $C_{L_{\alpha}} \%$ Difference & $C_{L_{0}}$ & $C_{L_{0}} \%$ Difference \\
\hline \hline UWAL Data & 4.0412 & 0 & 0.2251 & 0 \\
\hline VORLAX-FEA with reference VCCTEF & 3.7947 & -6.10 & 0.2294 & 1.91 \\
\hline VORLAX-FEA with mean VCCTEF & 3.7955 & -6.08 & 0.2374 & 5.51 \\
\hline
\end{tabular}

Table 18. Flexible UWAL Wind Tunnel Model Lift Curve Comparison, FLAP7 Cases

\section{FLAP8}

The FLAP8 configuration of the VCCTEF has the flaps deflected as a single rigid body flap where the second and third camber segments have zero relative deflection. Data collected with the FLAP8 configuration corresponds to runs 73 , 74, 75, 76, 77, and 78. The VCCTEF flap deflection data for these runs are presented in Table 19. 


\begin{tabular}{|c|c|c|c|c|c|c|c|c|c|c|c|c|c|c|c|}
\hline \multirow[b]{2}{*}{ Run } & \multicolumn{15}{|c|}{ VCCTEF Section/Segment Deflection } \\
\hline & $1 \mathrm{~A}$ & $1 \mathrm{~B}$ & $1 \mathrm{C}$ & $2 \mathrm{~A}$ & $2 \mathrm{~B}$ & $2 \mathrm{C}$ & $3 \mathrm{~A}$ & $3 \mathrm{~B}$ & $3 \mathrm{C}$ & $4 \mathrm{~A}$ & 4B & $4 \mathrm{C}$ & $5 \mathrm{~A}$ & $5 B$ & $5 \mathrm{C}$ \\
\hline Reference & 4.0 & $\mathbf{0 . 0}$ & $\mathbf{0 . 0}$ & 4.0 & 0.0 & 0.0 & 5.0 & $\mathbf{0 . 0}$ & $\mathbf{0 . 0}$ & 5.0 & $\mathbf{0 . 0}$ & $\mathbf{0 . 0}$ & 4.0 & 0.0 & $\mathbf{0 . 0}$ \\
\hline 73 & 2.1 & 0.9 & 0.8 & 3.8 & -0.4 & 4.1 & 3.1 & -0.4 & 3.8 & 2.5 & 1.2 & 2.5 & 2.5 & -0.8 & 2.5 \\
\hline 74 & 2.4 & 0.8 & 1.8 & 3.9 & 0.8 & 2.9 & 3.1 & -0.2 & 3.2 & 3.4 & 1.4 & 2.3 & 2.1 & 1.8 & -1.1 \\
\hline 75 & 2.5 & 0.6 & 2.8 & 2.7 & 2.4 & 1.8 & 3.3 & 0.8 & 2.6 & 3.6 & 1.5 & 2.1 & 2.3 & -0.5 & 1.7 \\
\hline 76 & 2.8 & 0.8 & 2.3 & 4.4 & 0.2 & 3.9 & 4.0 & 0.1 & 3.7 & 3.5 & 1.9 & 3.2 & 2.8 & -0.2 & 3.5 \\
\hline 77 & 3.2 & 0.9 & 2.3 & 5.5 & -0.2 & 4.6 & 4.6 & -1.0 & 6.0 & 4.5 & 1.5 & 3.4 & 2.4 & 2.3 & -1.8 \\
\hline 78 & 3.6 & 1.0 & 2.5 & 5.0 & 0.7 & 4.0 & 4.3 & 0.4 & 3.7 & 3.5 & 3.4 & 1.1 & 3.7 & -0.9 & 2.8 \\
\hline Mean & 2.77 & 0.83 & 2.08 & 4.17 & 0.58 & 3.55 & 3.73 & -0.05 & 3.83 & 3.5 & 1.82 & 2.43 & 2.63 & 0.28 & 1.27 \\
\hline
\end{tabular}

Table 19. VCCTEF Deflections and Corresponding Runs for FLAP8 Cases

The aeroelastic lift curves and drag polars from the UWAL test data are plotted with data from the VORLAX-FEA model. The results are shown in Fig. 27.
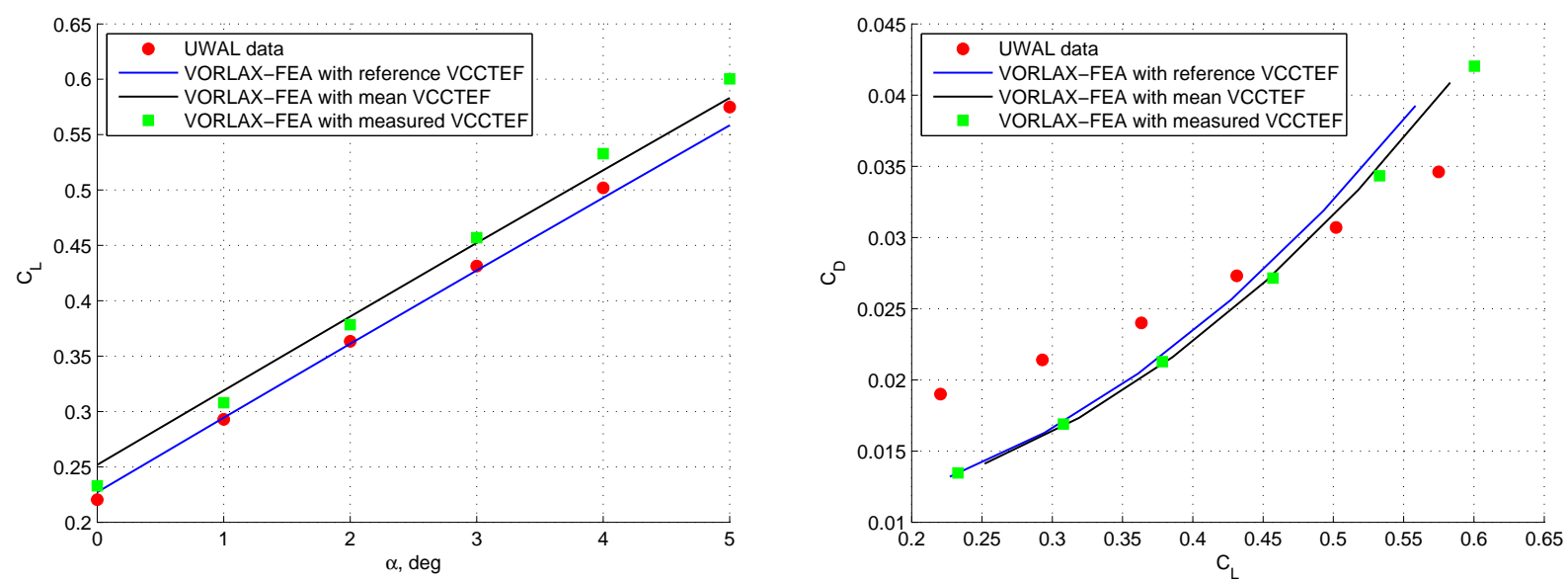

Figure 27. Flexible UWAL Wind Tunnel Model Lift Curve and Drag Polar for FLAP8 Cases

The parameters for the lift curve are shown in Table 20, and percent error values are calculated relative to the UWAL test data lift curve.

\begin{tabular}{|c|c|c|c|c|}
\hline & $C_{L_{\alpha}}$ & $C_{L_{\alpha}} \%$ Difference & $C_{L_{0}}$ & $C_{L_{0}} \%$ Difference \\
\hline \hline UWAL Data & 4.0392 & 0 & 0.2212 & 0 \\
\hline VORLAX-FEA with reference VCCTEF & 3.7965 & -6.01 & 0.2279 & 3.02 \\
\hline VORLAX-FEA with mean VCCTEF & 3.7972 & -5.99 & 0.2526 & 14.18 \\
\hline
\end{tabular}

Table 20. Flexible UWAL Wind Tunnel Model Lift Curve Comparison, FLAP8 Case

\section{J. FLAP9}

A flap case referred to as the FLAP9 configuration has deflection of only the third camber segments of the flaps. Data from runs $79,80,81,82,83$, and 84 correspond to the FLAP9 configuration, and the flap deflection data for each run is presented in Table 21 . 


\begin{tabular}{|c|c|c|c|c|c|c|c|c|c|c|c|c|c|c|c|}
\hline \multirow[b]{2}{*}{ Run } & \multicolumn{15}{|c|}{ VCCTEF Section/Segment Deflection } \\
\hline & $1 \mathrm{~A}$ & $1 \mathrm{~B}$ & $1 \mathrm{C}$ & $2 \mathrm{~A}$ & $2 \mathrm{~B}$ & $2 \mathrm{C}$ & $3 \mathrm{~A}$ & 3B & $3 \mathrm{C}$ & $4 \mathrm{~A}$ & 4B & $4 \mathrm{C}$ & $5 \mathrm{~A}$ & $5 \mathrm{~B}$ & $5 \mathrm{C}$ \\
\hline Reference & $\mathbf{0 . 0}$ & $\mathbf{0 . 0}$ & 6.0 & 0.0 & $\mathbf{0 . 0}$ & 6.0 & $\mathbf{0 . 0}$ & $\mathbf{0 . 0}$ & 8.0 & $\mathbf{0 . 0}$ & 0.0 & 6.0 & 0.0 & $\mathbf{0 . 0}$ & 6.0 \\
\hline 79 & 0.1 & 1.0 & 5.7 & 0.1 & 0.5 & 6.9 & 0.8 & -2.2 & 11.3 & -0.5 & 2.1 & 6.3 & -0.9 & 1.2 & 4.4 \\
\hline 80 & 0.5 & 0.7 & 6.0 & 1.9 & -1.4 & 9.2 & 0.6 & -0.9 & 8.9 & -1.1 & 3.3 & 5.5 & -0.8 & 3.1 & 0.2 \\
\hline 81 & 0.9 & 0.7 & 6.2 & 2.2 & -0.7 & 8.9 & 0.8 & -0.5 & 9.5 & 1.0 & 0.3 & 9.7 & 1.2 & -1.8 & 7.6 \\
\hline 82 & 1.2 & 1.0 & 6.9 & 1.9 & 0.1 & 8.6 & 0.9 & -0.9 & 10.6 & 1.4 & 1.3 & 8.6 & -0.5 & 2.8 & 1.1 \\
\hline 83 & 1.4 & 1.3 & 6.0 & 2.0 & -0.3 & 9.5 & 1.2 & 0.2 & 9.5 & 2.4 & 0.0 & 9.5 & 2.2 & -1.6 & 6.0 \\
\hline 84 & 1.6 & 1.5 & 6.4 & 2.4 & -0.2 & 9.5 & 1.6 & -0.7 & 11.0 & 2.2 & 0.7 & 9.8 & 1.1 & 1.0 & 5.0 \\
\hline Mean & 0.95 & 1.03 & 6.2 & 1.75 & -0.33 & 8.77 & 0.98 & -0.83 & 10.13 & 0.90 & 1.28 & 8.23 & 0.38 & 0.78 & 4.05 \\
\hline
\end{tabular}

Table 21. VCCTEF Deflections and Corresponding Runs for FLAP9 Cases

The lift curves and drag polars for the flexible UWAL model from the wind tunnel test as well as the VORLAXFEA aeroelastic model are shown in Fig. 28.
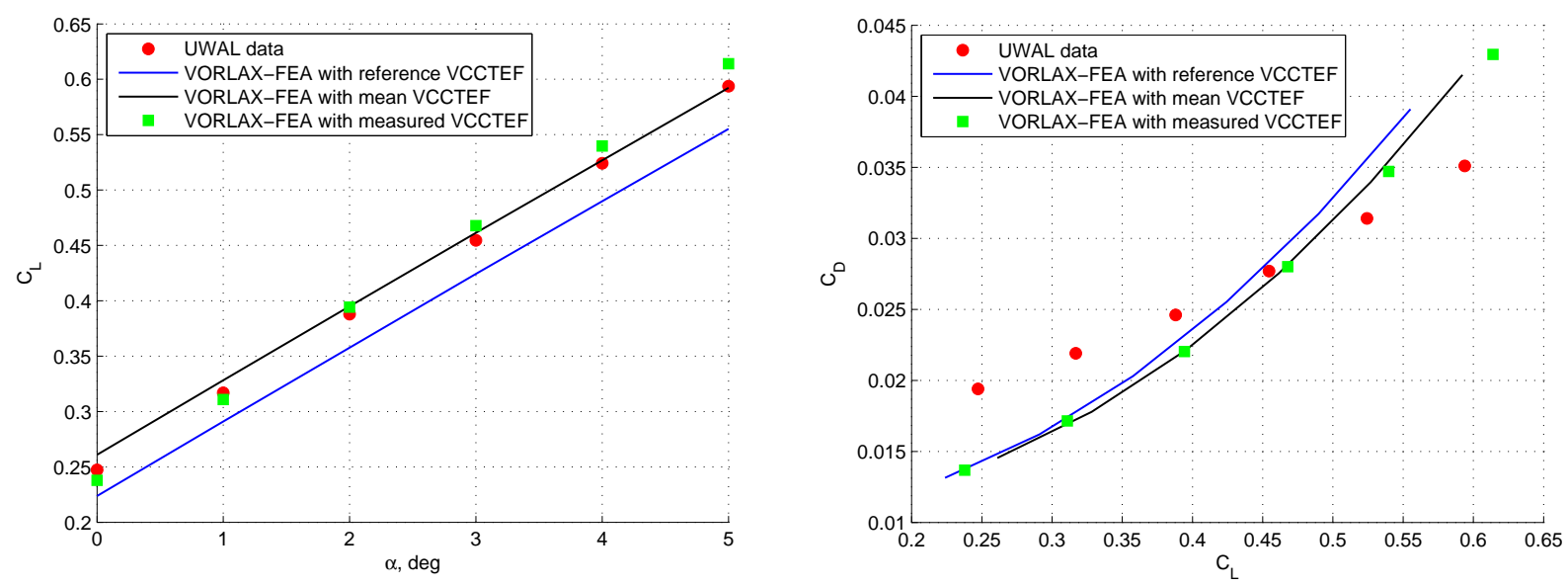

Figure 28. Flexible UWAL Wind Tunnel Model Lift Curve and Drag Polar for FLAP9 Cases

The values for the lift curve slope and zero angle of attack lift coefficient are tabulated in Table 22. Percent error values calculated relative to the UWAL data are included.

\begin{tabular}{|c|c|c|c|c|}
\hline & $C_{L_{\alpha}}$ & $C_{L_{\alpha}} \%$ Difference & $C_{L_{0}}$ & $C_{L_{0}} \%$ Difference \\
\hline \hline UWAL Data & 3.9631 & 0 & 0.2479 & 0 \\
\hline VORLAX-FEA with reference VCCTEF & 3.7982 & -4.16 & 0.2246 & -9.40 \\
\hline VORLAX-FEA with mean VCCTEF & 3.7982 & -4.23 & 0.2619 & 5.64 \\
\hline
\end{tabular}

Table 22. Flexible UWAL Wind Tunnel Model Lift Curve Comparison, FLAP9 Case

\section{K. FLAP10}

The FLAP10 configuration refers to runs $86,88,89,90,91$, and 92 where the flaps are deflected at positive "intermediate" values. The VCCTEF flap deflection data for each of the sections/segments are presented in Table 23. 


\begin{tabular}{|c|c|c|c|c|c|c|c|c|c|c|c|c|c|c|c|}
\hline \multirow[b]{2}{*}{ Run } & \multicolumn{15}{|c|}{ VCCTEF Section/Segment Deflection } \\
\hline & $1 \mathrm{~A}$ & 1B & $1 \mathrm{C}$ & $2 \mathrm{~A}$ & 2B & $2 \mathrm{C}$ & $3 \mathrm{~A}$ & $3 \mathrm{~B}$ & $3 \mathrm{C}$ & $4 \mathrm{~A}$ & 4B & $4 \mathrm{C}$ & $5 \mathrm{~A}$ & $5 \mathrm{~B}$ & $5 \mathrm{C}$ \\
\hline Reference & 2.0 & 3.0 & 2.0 & 3.0 & 3.0 & 1.0 & 4.0 & 2.0 & 5.0 & 2.0 & 5.0 & 2.0 & 2.0 & 5.0 & 3.0 \\
\hline 86 & 0.4 & 1.6 & 2.2 & 1.5 & 0.2 & 6.3 & 0.1 & 3.3 & 4.9 & 0.4 & 3.6 & 3.1 & 1.9 & 0.1 & 3.2 \\
\hline 88 & 0.6 & 1.7 & 1.9 & 0.9 & 1.4 & 4.5 & 0.6 & 1.0 & 8.2 & 1.0 & 3.1 & 3.3 & 0.5 & 3.0 & 0.4 \\
\hline 89 & 1.0 & 2.0 & 1.7 & 2.9 & -0.2 & 6.1 & 1.0 & 2.3 & 6.1 & 1.4 & 3.1 & 2.7 & 1.8 & -0.3 & 3.2 \\
\hline 90 & 1.2 & 2.3 & 2.3 & 2.8 & 1.2 & 5.2 & 1.9 & 1.7 & 7.0 & 1.8 & 2.6 & 5.0 & 2.9 & -1.5 & 7.1 \\
\hline 91 & 1.3 & 2.3 & 2.4 & 2.8 & 1.5 & 5.4 & 1.6 & 1.7 & 8.1 & 2.7 & 2.1 & 5.7 & 1.2 & 3.0 & 0.6 \\
\hline 92 & 1.5 & 2.6 & 2.4 & 3.1 & 0.5 & 7.0 & 1.7 & 2.9 & 7.2 & 3.2 & 2.3 & 5.2 & 2.2 & 1.3 & 2.3 \\
\hline Mean & 1.00 & 2.08 & 2.15 & 2.33 & 0.77 & 5.75 & 1.15 & 2.15 & 6.92 & 1.75 & 2.80 & 4.17 & 1.75 & 0.93 & 2.80 \\
\hline
\end{tabular}

Table 23. VCCTEF Deflections and Corresponding Runs for FLAP10 Cases

Flexible UWAL wind tunnel model lift curves and drag polars generated using the VORLAX-FEA are shown in Fig. 29 against the wind tunnel test data.
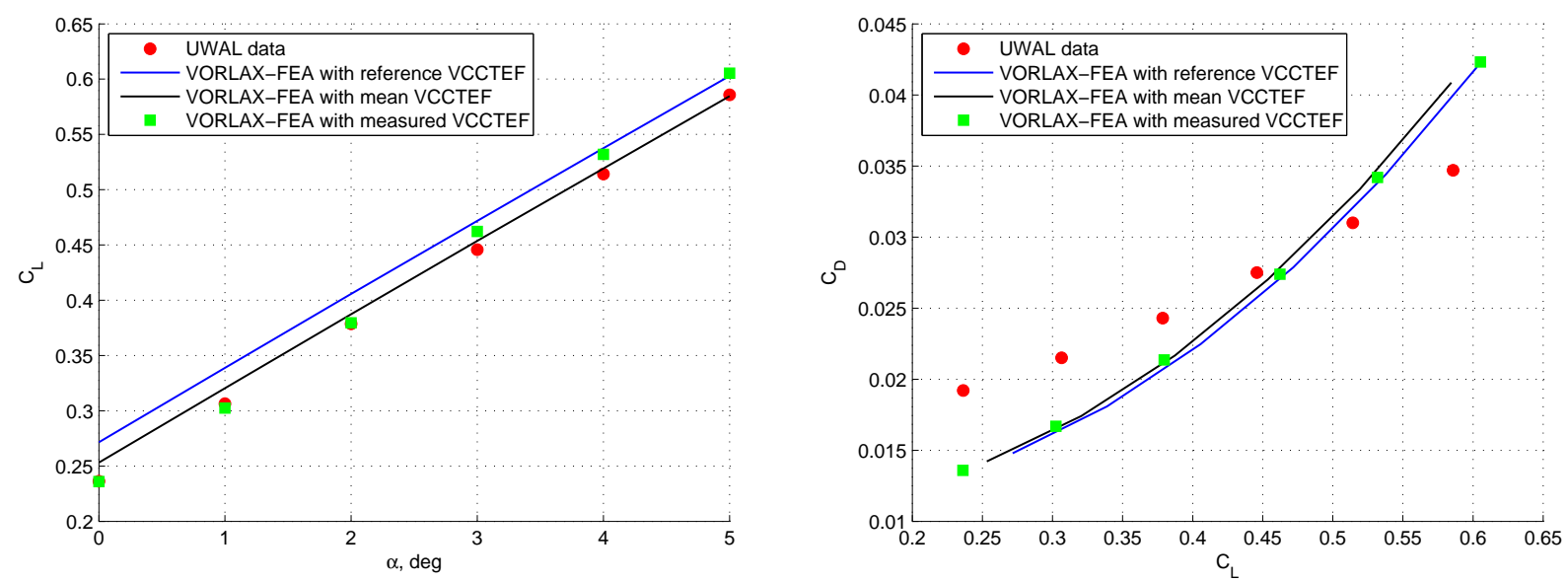

Figure 29. Flexible UWAL Wind Tunnel Model Lift Curve and Drag Polar for FLAP10 Cases

Table 24 summarizes the lift curve parameters from the aeroelastic model and the percent error values relative to the UWAL data.

\begin{tabular}{|c|c|c|c|c|}
\hline & $C_{L_{\alpha}}$ & $C_{L_{\alpha}} \%$ Difference & $C_{L_{0}}$ & $C_{L_{0}} \%$ Difference \\
\hline \hline UWAL Data & 3.9912 & 0 & 0.2370 & 0 \\
\hline VORLAX-FEA with reference VCCTEF & 3.7964 & -4.88 & 0.2724 & 14.92 \\
\hline VORLAX-FEA with mean VCCTEF & 3.7978 & -4.85 & 0.2540 & 7.16 \\
\hline
\end{tabular}

Table 24. Flexible UWAL Wind Tunnel Model Lift Curve Comparison, FLAP10 Cases

\section{FLAP11}

The final case examined in this study corresponds to the FLAP11 configuration where the flaps are deflected fully in the negative direction. The data for runs 93, 94, 95, 96, 97, 98, 99, and 100 correspond to data collected at the FLAP11 configuration. Table 25 summarizes the VICON measured VCCTEF flap deflections as well the reference setting. 


\begin{tabular}{|c|c|c|c|c|c|c|c|c|c|c|c|c|c|c|c|}
\hline \multirow[b]{2}{*}{ Run } & \multicolumn{15}{|c|}{ VCCTEF Section/Segment Deflection } \\
\hline & $1 \mathrm{~A}$ & $1 \mathrm{~B}$ & $1 \mathrm{C}$ & $2 \mathrm{~A}$ & 2B & $2 \mathrm{C}$ & $3 \mathrm{~A}$ & 3B & $3 \mathrm{C}$ & $4 \mathrm{~A}$ & 4B & $4 \mathrm{C}$ & $5 \mathrm{~A}$ & $5 \mathrm{~B}$ & $5 \mathrm{C}$ \\
\hline Reference & -1.0 & $\mathbf{0 . 0}$ & -3.0 & $\mathbf{0 . 0}$ & $\mathbf{0 . 0}$ & -6.0 & -1.0 & -4.0 & $\mathbf{0 . 0}$ & -2.0 & $\mathbf{0 . 0}$ & -5.0 & -4.0 & $\mathbf{0 . 0}$ & -4.0 \\
\hline 93 & -0.3 & 0.1 & -3.7 & 0.2 & -0.8 & -3.5 & -0.8 & -2.0 & -1.1 & -2.3 & 2.1 & -7.3 & -0.1 & -1.3 & -3.7 \\
\hline 94 & -0.1 & 0.3 & -3.6 & 0.8 & -1.3 & -2.4 & 0.1 & -3.3 & 0.0 & 0.2 & -1.2 & -3.5 & 0.2 & 0.3 & -5.8 \\
\hline 95 & -0.3 & 0.2 & -3.3 & 0.4 & -0.1 & -3.1 & 0.8 & -3.2 & 0.2 & -0.9 & 2.0 & -6.6 & 0.5 & -0.7 & -4.1 \\
\hline 96 & -0.8 & 0.0 & -2.3 & 1.4 & -0.7 & -2.5 & 0.2 & -1.5 & -0.7 & 0.2 & 0.2 & -3.5 & 2.2 & -3.2 & -0.9 \\
\hline 97 & 1.3 & 0.0 & -2.0 & 2.1 & -1.8 & -0.8 & 1.6 & -3.0 & 1.4 & 0.3 & 0.9 & -4.3 & 0.4 & 2.7 & -8.2 \\
\hline 98 & 1.4 & 0.2 & -2.2 & 2.3 & -1.4 & -1.1 & 1.8 & -2.7 & 0.4 & 1.3 & -0.2 & -2.1 & 2.5 & -0.7 & -3.7 \\
\hline 99 & 1.6 & 0.3 & -2.2 & 2.0 & 0.1 & -2.1 & 2.2 & -2.8 & 2.2 & 1.1 & 1.5 & -3.7 & 1.0 & 0.1 & -3.7 \\
\hline 100 & 1.8 & 0.2 & -1.6 & 2.5 & -0.2 & -1.7 & 1.7 & -0.7 & -0.5 & 2.2 & -0.4 & -1.2 & 2.2 & -1.80 & -2.3 \\
\hline Mean & 0.85 & 0.16 & -2.61 & 1.46 & -0.78 & -2.15 & 0.95 & -2.23 & 0.24 & 0.26 & 0.61 & -4.03 & 1.11 & -0.58 & -4.05 \\
\hline
\end{tabular}

Table 25. VCCTEF Deflections and Corresponding Runs for FLAP11 Cases

The lift curve and drag polar plots are shown in Fig. 30 which includes the wind tunnel test data in comparison to the results from the VORLAX-FEA model.
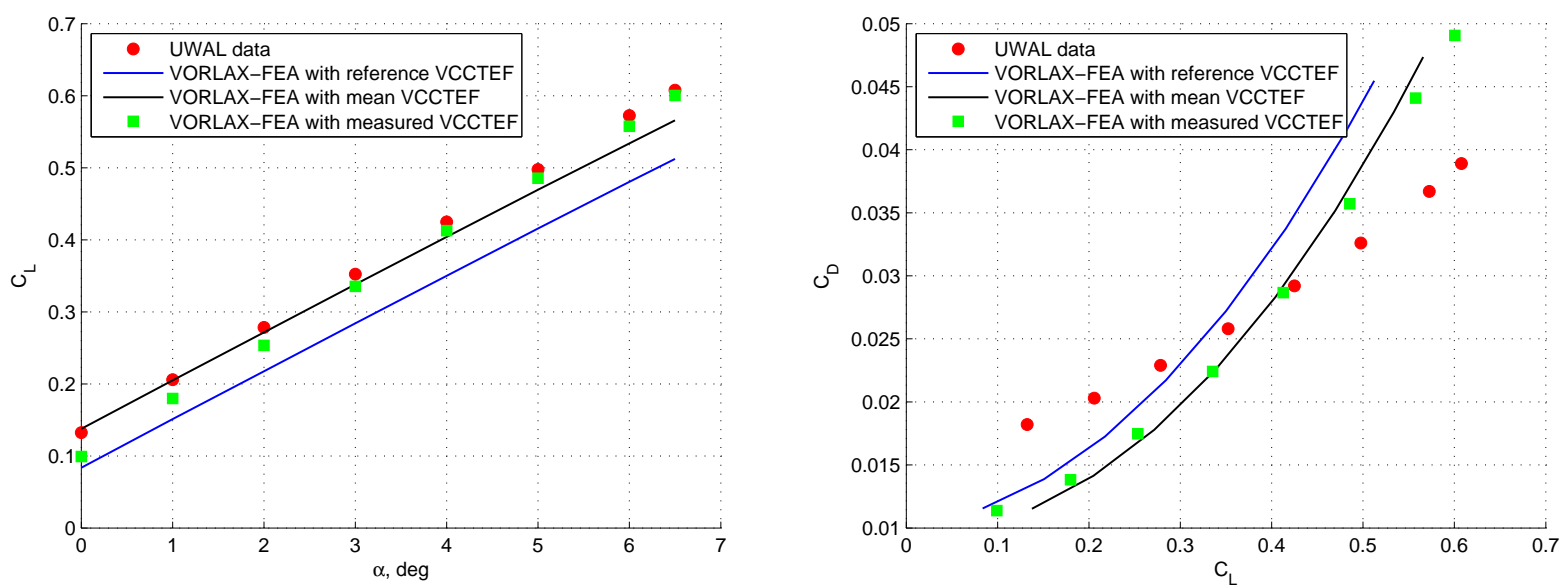

Figure 30. Flexible UWAL Wind Tunnel Model Lift Curve and Drag Polar for FLAP11 Cases

The lift curve parameters describing the data shown in Fig. 30 are summarized in Table 26.

\begin{tabular}{|c|c|c|c|c|}
\hline & $C_{L_{\alpha}}$ & $C_{L_{\alpha}} \%$ Difference & $C_{L_{0}}$ & $C_{L_{0}} \%$ Difference \\
\hline \hline UWAL Data & 4.1933 & 0 & 0.1324 & 0 \\
\hline VORLAX-FEA with reference VCCTEF & 3.7792 & -9.87 & 0.0851 & -35.71 \\
\hline VORLAX-FEA with mean VCCTEF & 3.7750 & -9.97 & 0.1392 & 5.12 \\
\hline
\end{tabular}

Table 26. Flexible UWAL Wind Tunnel Model Lift Curve Comparison, FLAP11 Cases

\section{Summary of Lift Curve Comparisons}

The percentage error values between the VORLAX-FEA lift curves relative to the UWAL wind tunnel test data for the FLAP0-FLAP11 comparisons completed are re-summarized in Table 27. 


\begin{tabular}{|c|c|c|c|c|}
\hline & \multicolumn{2}{|c|}{ VORLAX-FEA with reference VCCTEF } & \multicolumn{2}{|c|}{ VORLAX-FEA with mean VCCTEF } \\
\hline FLAP setting & $C_{L_{\alpha}} \%$ Difference & $C_{L_{0}} \%$ Difference & $C_{L_{\alpha}} \%$ Difference & $C_{L_{0}} \%$ Difference \\
\hline \hline FLAP0 & -7.93 & -12.84 & -7.71 & 3.26 \\
\hline FLAP1 & 3.53 & 24.19 & 3.74 & 12.52 \\
\hline FLAP2 & 0.01 & 17.44 & -0.09 & 3.66 \\
\hline FLAP3 & 1.55 & 19.12 & 1.82 & 9.45 \\
\hline FLAP4 & -1.67 & 11.99 & -1.56 & 5.87 \\
\hline FLAP5 & -3.77 & 2.70 & -3.80 & 8.69 \\
\hline FLAP6 & -7.16 & -10.89 & -7.17 & 5.21 \\
\hline FLAP7 & -6.10 & 1.91 & -6.08 & 5.51 \\
\hline FLAP8 & -6.01 & 3.02 & -5.99 & 14.18 \\
\hline FLAP9 & -4.16 & -9.40 & -4.23 & 5.64 \\
\hline FLAP10 & -4.88 & 14.92 & -4.85 & 7.16 \\
\hline FLAP11 & -9.87 & -35.71 & -9.97 & 5.12 \\
\hline
\end{tabular}

Table 27. Summary of Percent Difference in Aeroelastic Lift Curve Parameters Relative to UWAL test data.

The data from Table 27 shows that the VORLAX-FEA model is able to predict the $C_{L_{\alpha}}$ or lift curve slope of the different runs of the flexible UWAL wind tunnel model very well. Using the reference VCCTEF settings, a maximum magnitude of $9.87 \%$ error exists for the FLAP11 configuration. A maximum magnitude of $9.97 \%$ error corresponds to the difference between the VORLAX-FEA model and the actual wind tunnel data when using the mean VCCTEF deflections as measured from the VICON data. In terms of $C_{L_{0}}$ or the lift coefficient at zero angle of attack, using the reference VCCTEF settings in the aeroelastic model generates values that have a maximum discrepancy of $35.71 \%$ also occurring in the FLAP11 data set analysis. Using mean VCCTEF values calculated from VICON measurements seem to improve the $C_{L_{0}}$ estimate for most of the flap configurations, decreases the maximum discrepancy down to a magnitude of $14.18 \%$.

\section{Conclusion}

This paper presents the static aeroelastic modeling effort of a flexible sub-scale model of a wing with VCCTEF control effector. The physical model is sub-scale version of the GTM wing which was constructed and wind tunnel tested at UWAL as a proof-of-concept test of the VCCTEF design. The static aeroelastic model is built using a coupled vortex-lattice finite-element approach. The vortex-lattice code VORLAX is used as the aerodynamic model, and a finite-element modeling of an equivalent beam structural model is detailed. An automated geometry generation tool is used in order to enable coupled aero-structural iterations by generating VLM input geometry. The resulting aeroelastic model is referred to as a VORLAX-FEA model and is compared against a VORLAX-NASTRAN model where the structural model is replaced by an identical beam set-up run with the commercial NASTRAN code. A strong agreement between aeroelastic deformations calculated from the VORLAX-FEA model and the VORLAX-NASTRAN model $(<1 \%$ error in deformation values) presents validation of the finite-element framework implemented in the VORLAXFEA aeroelastic model. The static aeroelastic model is used to predict the aerodynamics of the flexible UWAL model at given flight conditions.

The automated geometry generation tool utilized by the static aeroelastic model also facilitates modeling the deflection of the VCCTEF flap sections of the model. The UWAL wind tunnel model possesses a VCCTEF with five flap sections with three camber segments per flap. Data from the UWAL wind tunnel test in 2013 resulted in a data package that included 125 useable data runs of the flexible aeroelastic model at various test conditions and flap settings. The static aeroelastic VORLAX-FEA is run and compared against the UWAL data results as a validation study. A total of a dozen comparison data sets are analyzed in this study, corresponding to reference flap settings identified as FLAP0 through FLAP11. Based on reference flap settings, the VORLAX-FEA code is used to generate aeroelastic lift-curves and drag polars to compare against wind tunnel results. A maximum magnitude percent difference of 9.87\% was observed between the lift curve slope values $C_{L_{\alpha}}$ calculated using VORLAX-FEA and the wind tunnel results. A maximum magnitude percent difference of $35.71 \%$ was observed in the prediction of zero angle of attack 
lift coefficient from VORLAX-FEA and wind tunnel data. Drag polar data was also presented from the results, but drag prediction is known to be notoriously hard. Despite incorporating a skin friction drag correction to induced drag predictions from vortex-lattice, unknown imperfections in the model and unmodeled effects, including the effect of VCCTEF deflections on parasite drag, limit the drag prediction capability of the developed static aeroelastic model.

The UWAL wind tunnel data utilized a VICON motion tracking system in order to conduct measurements of the model's deformed geometry. It was observed that during the tests, while wind on, the VCCTEF flap sections deviated from the reference values that they were set at due to the nature of the construction of the model. VICON data is available that measures the VCCTEF actual deflection during the test for the 12 data sets analyzed in this study. The VICON measured deflection shows very high deviation and spread from the reference VCCTEF settings. Though VICON data is prone to inaccuracies, the VICON measured VCCTEF flap deflection data is averaged for each of the data set and VORLAX-FEA is run using the mean VCCTEF flap deflections to obtain updated aeroelastic lift-curves and drag polars. Using mean deflections as measured by the VICON system, the agreement in lift curve slope from the aeroelastic VORLAX-FEA model retained a strong agreement with wind tunnel data: a maximum magnitude of 9.97\% error. The disagreement between zero angle of attack lift coefficient was also improved to a maximum percent difference of $14.18 \%$.

From the tables presented in each of the individual flap data set comparisons, a wide variation is observed between the VICON measured data and the reference flap settings. The spread of the VCCTEF measurements propagates into the lift curve figures presented, where the data points corresponding to the aeroelastic model runs with measured deflections are generally scattered. The data indicates the deficiency of the sub-scale model's construction in being able to fix the VCCTEF flap settings, which deviate significantly during the tests. Since VCCTEF deflections affect $C_{L_{0}}$, these deviations in flap settings as well as inaccuracies of the VICON system measurements present a significant possible contributor to error between the VORLAX-FEA model and the UWAL test data for $C_{L_{0}}$. Despite taking the average of the VICON measured VCCTEF data, there is no way to accurately know the VCCTEF deflections with certainty. It was also advised from UWAL that the construction of the wind tunnel model introduced a degree of uncertainty in the wing incidence angle at the root. Since small angle changes in the wing incidence angle can impact $C_{L_{0}}$, it is realized that correct prediction of zero angle of attack lift coefficient is very difficult of the UWAL model. Despite relatively larger percent errors in values of $C_{L_{0}}$, however, the lift curve slope $C_{L_{\alpha}}$ is predicted quite well by the static aeroelastic model. In terms of aeroelastic modeling, $C_{L_{\alpha}}$ is mainly impacted by the aeroelastic wash-out twist due to bending. The strong percent agreement between the static aeroelastic model and wind tunnel data values for $C_{L_{\alpha}}$ validates the aeroelastic predictive capability of the model.

\section{Acknowledgments}

The authors would like to thank the Fixed Wing Project under the Fundamental Aeronautics Program of NASA Aeronautics Research Mission Directorate (ARMD) for funding support of this work. The authors also would like to acknowledge Boeing Research and Technology and the University of Washington Aeronautical Laboratory for their collaboration with NASA on this work.

\section{References}

\footnotetext{
${ }^{1}$ Nguyen, N., "Elastically Shaped Future Air Vehicle Concept," NASA Innovation Fund Award 2010 Report, http://ntrs.nasa.gov/archive/nasa/casi.ntrs.nasa.gov/20110023698_2011024909.pdf, Submitted to NASA Innovative Partnerships Program, October 2010.

${ }^{2}$ Nguyen, N., Trinh, K., Reynolds, K., Kless, J., Aftosmis, M., Urnes, J., and Ippolito C., "Elastically Shaped Wing Optimization and Aircraft Concept for Improved Cruise Efficiency," AIAA Aerospace Sciences Meeting, AIAA-2013-0141, January 2013.

${ }^{3}$ Boeing Report No. 2010X0015, "Development of Variable Camber Continuous Trailing Edge Flap System," October 4, 2012.

${ }^{4}$ Urnes, J., Nguyen N., Ippolito, C., Totah, J., Trinh, K., and Ting, E., “A Mission Adaptive Variable Camber Flap Control System to Optimize High Lift and Cruise Lift to Drag Ratios of Future N+3 Transport Aircraft," AIAA Aerospace Sciences meeting, AIAA-2013-0214, January 2013.

${ }^{5}$ Jordan, T. L., Langford, W. M., Belcastro, C. M., Foster, J. M., Shah, G. H., Howland, G., and Kidd, R., "Development of a Dynamically Scaled Generic Transport Model Testbed for Flight Research Experiments," AUVSI Unmanned Unlimited, Arlington, VA, 2004.

${ }^{6}$ Precup, N., Mor, M., Livne, E., "Design, Construction, and Tests of an Aeroelastic Wind Tunnel Model of a Variable Camber Continuous Trailing Edge Flap (VCCTEF) Concept Wing," AIAA Applied Aerodynamics Conference, AIAA-2014-2442, June 2014.

${ }^{7}$ Nguyen, N., Precup, N., Urnes, J., Nelson, C., Lebofsky, S., Ting, E., Livne, E., "Experimental Investigation of a Flexible Wing with a Variable Camber Continuous Trailing Edge Flap Design," AIAA Applied Aerodynamics Conference, AIAA 2014-2441, June 2014.

${ }^{8}$ Rodriguez, D., Aftosmis, M., Nemec, M., Smith, S., "Static Aeroelastic Analysis with an Invisicid Cartesian Method," AIAA Structures, Structural Dynamics, and Materials Conference, AIAA 2014-0836, January 2014.
} 
${ }^{9}$ Craig, Jr., R.R. and Kurdila, A.J., Fundamentals of Structural Dynamics, Second Edition, John Wiley \& Sons, Inc., 2006.

${ }^{10}$ Hodges, D.H. and Pierce, G.A., Introduction to Structural Dynamics and Aeroelasticity, Cambridge University Press, 2002.

${ }^{11}$ Hughes, T., The Finite Element Method Linear Static and Dynamic Finite Element Analysis, Prentice Hall, Inc., 1987.

${ }^{12}$ Meirovitch, L., Fundamentals of Vibrations, McGraw-Hill Higher Education, 2001.

${ }^{13}$ Nguyen, N., Ting, E., Lebofsky, S., "Aeroelastic Analysis of a Flexible Wing Wind Tunnel Model with Variable Camber Continuous Trailing Edge Flap Design", To Be Presented at AIAA Structures, Structural Dynamics, and Materials Conference, January 2015.

${ }^{14}$ Miranda, L.R., Elliot, R.D., and Baker, W.M., "A Generalized Vortex Lattice Method for Subsonic and Supersonic Flow Applications," NASA CR-2865, 1977.

${ }^{15}$ Nguyen, N., Trinh, K., Frost S., and Reynolds, K., "Coupled Aeroelastic Vortex Lattice Modeling of Flexible Aircraft," AIAA Applied Aerodynamics Conference, AIAA-2011-3021, June 2011.

${ }^{16}$ Nguyen, N., Trinh, K., Nguyen, D., Tuzcu, I., "Nonlinear Aeroelasticty of Flexible Wing Structure Coupled with Aircraft Flight Dynamics," AIAA Structures, Structural Dynamics, and Materials Conference, AIAA-2012-1792, April 2012.

${ }^{17}$ Nguyen, N., Ting, E., Nguyen, D., Dao, T., Trinh, K., "Coupled Vortex-Lattice Flight Dynamic Model with Aeroelastic Finite-Element Model of Flexible Wing Transport Aircraft with Variable Camber Continuous Trailing Edge Flap for Drag Reduction", AIAA Atmospheric Flight Mechanics (AFM) Conference, AIAA-2013-4746, August 2013

${ }^{18}$ Ting, E., Nguyen, N., Trinh, K., "Static Aeroelastic and Longitudinal Trim Model of Flexible Wing Aircraft Using Finite-Element VortexLattice Coupled Solution", AIAA Structures, Structural Dynamics, and Materials Conference, AIAA-2014-0837, January 2014.

${ }^{19}$ Ting, E., Lebofsky, S., Nguyen, N., Trinh, K., "Static Aeroelastic Scaling and Analysis of a Sub-Scale Flexible Wing Wind Tunnel Model", AIAA Structures, Structural Dynamics, and Materials Conference, AIAA-2014-0838, January 2014.

${ }^{20}$ Lebofsky, S., Ting, E., Nguyen, N., "Aeroelastic Modeling and Drag Optimization of Aircraft Wing with Variable Camber Continuous Trailing Edge Flap," AIAA Applied Aerodynamics Conference, AIAA-2014-2443, June 2014.

${ }^{21}$ Aftosmis, M.J., Berger, M.J., and Melton, J.E., "Robust and Efficient CartesianMesh Generation for Component- Based Geometry," AIAA Journal, Vol. 36, No. 6, 1998, pp. 953-960.

${ }^{22}$ Houbolt, J. C and Brooks, G. W., "Differential Equations of Motion for Combined Flapwise Bending, Chordwise Bending, and Torsion of Twisted Nonuniform Rotor Blades," NACA Technical Note 3905, February 1957. 